Cochrane Database of Systematic Reviews

\title{
Head midline position for preventing the occurrence or extension of germinal matrix-intraventricular hemorrhage in preterm infants (Review)
}

Romantsik O, Calevo MG, Bruschettini M

Romantsik O, Calevo MG, Bruschettini M.

Head midline position for preventing the occurrence or extension of germinal matrix-intraventricular hemorrhage in preterm infants. Cochrane Database of Systematic Reviews 2017, Issue 7. Art. No.: CD012362.

DOI: 10.1002/14651858.CD012362.pub2.

www.cochranelibrary.com

Head midline position for preventing the occurrence or extension of germinal matrix-intraventricular hemorrhage in preterm infants (Review) 


\section{TABLE OF CONTENTS}

HEADER

ABSTRACT

PLAIN LANGUAGE SUMMARY

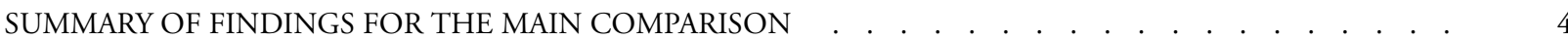

BACKGROUND . . . . . . . . . . . . . . . . . . . . . . . . . . . . . . . . . . . . . 7

OBJECTIVES . . . . . . . . . . . . . . . . . . . . . . . . . . . . . . . . . . . . . 8

METHODS . . . . . . . . . . . . . . . . . . . . . . . . . . . . . . . . . . . . . .

RESULTS . . . . . . . . . . . . . . . . . . . . . . . . . . . . . . . . . . 13

Figure 1. . . . . . . . . . . . . . . . . . . . . . . . . . . . . . . . . . . . . 14

Figure 2. . . . . . . . . . . . . . . . . . . . . . . . . . . . . . . . . . . . . .

Figure $3 . \quad$. . . . . . . . . . . . . . . . . . . . . . . . . . . . . . . . . . . . . . . . . . .

Figure $4 . \quad$. . . . . . . . . . . . . . . . . . . . . . . . . . . . . . . . . . . . . 18

Figure 5. . . . . . . . . . . . . . . . . . . . . . . . . . . . . . . . . . . . . . 19

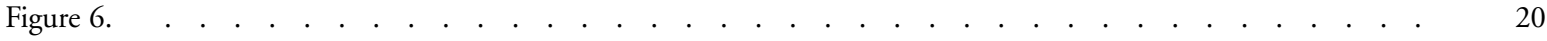

DISCUSSION . . . . . . . . . . . . . . . . . . . . . . . . . . . . . . . . . . . 21

AUTHORS' CONCLUSIONS . . . . . . . . . . . . . . . . . . . . . . . . . . . . . . . . 22

ACKNOWLEDGEMENTS . . . . . . . . . . . . . . . . . . . . . . . . . . . . . . . . . . . 22

REFERENCES . . . . . . . . . . . . . . . . . . . . . . . . . . . . . . . . . . . . . . . . . . 22

CHARACTERISTICS OF STUDIES . . . . . . . . . . . . . . . . . . . . . . . . . . . . . . . . . . . . .

DATA AND ANALYSES . . . . . . . . . . . . . . . . . . . . . . . . . . . . . . . . . . . . . . . . . . . . . . . . . $\quad . \quad 29$

Analysis 1.1. Comparison 1 Supine midline head position with the bed at $0^{\circ}$ versus supine head rotated $90^{\circ}$ with the bed at

$0^{\circ}$, Outcome 1 Intraventricular hemorrhage, any grade. . . . . . . . . . . . . . . . . . . . 29

Analysis 1.2. Comparison 1 Supine midline head position with the bed at $0^{\circ}$ versus supine head rotated $90^{\circ}$ with the bed at $0^{\circ}$, Outcome 2 Intraventricular hemorrhage, grade 3 to $4 . \quad$. . . . . . . . . . . . . . . . . . . . . . . 30

Analysis 1.3. Comparison 1 Supine midline head position with the bed at $0^{\circ}$ versus supine head rotated $90^{\circ}$ with the bed at $0^{\circ}$, Outcome 3 Neonatal mortality. . . . . . . . . . . . . . . . . . . . . . . . . . . 30

Analysis 1.4. Comparison 1 Supine midline head position with the bed at $0^{\circ}$ versus supine head rotated $90^{\circ}$ with the bed at $0^{\circ}$, Outcome 4 Cystic periventricular leukomalacia. . . . . . . . . . . . . . . . . . . . . . 31

Analysis 1.5. Comparison 1 Supine midline head position with the bed at $0^{\circ}$ versus supine head rotated $90^{\circ}$ with the bed at $0^{\circ}$, Outcome 5 Retinopathy of prematurity, any stage. . . . . . . . . . . . . . . . . . . . . . . . . 32

Analysis 1.6. Comparison 1 Supine midline head position with the bed at $0^{\circ}$ versus supine head rotated $90^{\circ}$ with the bed at $0^{\circ}$, Outcome 6 Retinopathy of prematurity, $\geq$ stage 3. . . . . . . . . . . . . . . . . . . . . . . . 32

APPENDICES . . . . . . . . . . . . . . . . . . . . . . . . . . . . . . . . . . . . . 32

CONTRIBUTIONS OF AUTHORS . . . . . . . . . . . . . . . . . . . . . . . . . . . . . . . . . . . . . . .

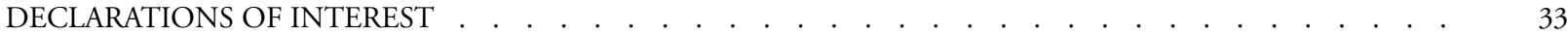

SOURCES OF SUPPORT . . . . . . . . . . . . . . . . . . . . . . . . . . . . . . . . . . . . . . . . . . . .

INDEX TERMS . . . . . . . . . . . . . . . . . . . . . . . . . . . . . . . . . . . . . 33

Head midline position for preventing the occurrence or extension of germinal matrix-intraventricular hemorrhage in preterm infants 
[Intervention Review]

\title{
Head midline position for preventing the occurrence or extension of germinal matrix-intraventricular hemorrhage in preterm infants
}

\author{
Olga Romantsik ${ }^{1}$, Maria Grazia Calevo ${ }^{2}$, Matteo Bruschettini ${ }^{1,3}$ \\ ${ }^{1}$ Department of Paediatrics, Lund University, Skåne University Hospital, Lund, Sweden. ${ }^{2}$ Epidemiology, Biostatistics and Committees \\ Unit, Istituto Giannina Gaslini, Genoa, Italy. ${ }^{3}$ Research \& Development, Section for HTA Analysis, Skåne University Hospital, Lund, \\ Sweden \\ Contact address: Matteo Bruschettini, Department of Paediatrics, Lund University, Skåne University Hospital, Lund, Sweden. \\ matteo.bruschettini@med.lu.se, matbrus@gmail.com.
}

Editorial group: Cochrane Neonatal Group.

Publication status and date: New, published in Issue 7, 2017.

Citation: Romantsik O, Calevo MG, Bruschettini M. Head midline position for preventing the occurrence or extension of germinal matrix-intraventricular hemorrhage in preterm infants. Cochrane Database of Systematic Reviews 2017, Issue 7. Art. No.: CD012362. DOI: 10.1002/14651858.CD012362.pub2.

Copyright (C) 2017 The Cochrane Collaboration. Published by John Wiley \& Sons, Ltd.

\section{A B S T R A C T}

\section{Background}

Preterm birth is known to constitute the major risk factor for development of germinal matrix-intraventricular hemorrhage (GMIVH). Head position may affect cerebral hemodynamics and thus may be involved indirectly in development of GM-IVH. Turning the head toward one side may functionally occlude jugular venous drainage on the ipsilateral side while increasing intracranial pressure and cerebral blood volume. Thus, it has been suggested that cerebral venous pressure is reduced and hydrostatic brain drainage improved if the patient is in supine midline position with the bed tilted $30^{\circ}$. The midline position might be achieved in the supine position and, with the use of physical aids, in the lateral position as well. Midline position should be kept, at least when the incidence of GM-IVH is greatest, that is, during the first two to three days of life.

\section{Objectives}

\section{Primary objective}

To assess whether head midline position is more effective than any other head position for preventing or extending germinal matrixintraventricular hemorrhage in infants born at $\leq 32$ weeks' gestational age.

\section{Secondary objectives}

To perform subgroup analyses regarding gestational age, birth weight, intubated versus not intubated, and with or without GM-IVH at trial entry.

\section{Search methods}

We used the standard search strategy of the Cochrane Neonatal Review Group to search the Cochrane Central Register of Controlled Trials (CENTRAL; 2016, Issue 8), MEDLINE via PubMed (1966 to September 19, 2016), Embase (1980 to September 19,.2016), and the Cumulative Index to Nursing and Allied Health Literature (CINAHL; 1982 to September 19, 2016). We searched clinical trials databases, conference proceedings, and reference lists of retrieved articles for randomized controlled trials and quasi-randomized trials.

Head midline position for preventing the occurrence or extension of germinal matrix-intraventricular hemorrhage in preterm infants 


\section{Selection criteria}

Randomized clinical controlled trials, quasi-randomized trials, and cluster-randomized controlled trials comparing placing very preterm infants in a head midline position versus placing them in a prone or lateral decubitus position, or undertaking a strategy of regular position change, or having no prespecified position. We included trials enrolling infants with existing GM-IVH and planned to assess extension of hemorrhage in a subgroup of infants. We planned to analyze horizontal (flat) versus head elevated positions separately for all body positions.

\section{Data collection and analysis}

We used standard methods of the Cochrane Neonatal Review Group. For each of the included trials, two review authors independently extracted data (e.g., number of participants, birth weight, gestational age, initiation and duration of head midline position, cointervention with horizontal vs head elevated position, use of physical aids to maintain head position) and assessed risk of bias (e.g., adequacy of randomization, blinding, completeness of follow-up). The primary outcomes considered in this review are GM-IVH , severe IVH, and neonatal death.

\section{Main results}

Our search strategy yielded 2696 references. Two review authors independently assessed all references for inclusion. Two randomized controlled trials, for a total of 110 infants, met the inclusion criteria of this review. Both trials compared supine midline head position with the bed at $0^{\circ}$ versus supine head rotated $90^{\circ}$ with the bed at $0^{\circ}$. We found no trials that compared supine versus prone midline head position, and no trials that compared effects of head tilting. We found no significant differences in rates of GM-IVH (typical risk ratio $[R R] 1.14,95 \%$ confidence interval [CI] 0.55 to 2.35 ; typical risk difference [RD] 0.03 , 95\% CI -0.13 to 0.18 ; two studies, 110 infants; $\mathrm{I}^{2}=0 \%$ for RR and $\mathrm{I}^{2}=0 \%$ for RD), severe IVH (typical RR $1.57,95 \%$ CI 0.28 to 8.98 ; typical RD $0.02,95 \%$ CI -0.06 to 0.10 ; two studies, 110 infants; $\mathrm{I}^{2}=0 \%$ for $\mathrm{RR}$ and $\mathrm{I}^{2}=0 \%$ for $\mathrm{RD}$ ), and neonatal mortality (typical RR $0.52,95 \% \mathrm{CI}$ 0.16 to 1.65 ; typical RD $-0.07,95 \%$ CI -0.18 to 0.05 ; two studies, 110 infants; $I^{2}=28 \%$ for RR and $I^{2}=44 \%$ for RD). Among secondary outcomes, we found no significant differences in terms of cystic periventricular leukomalacia (one study; RR 3.25, 95\% CI 0.14 to 76.01 ; RD $0.04,95 \%$ CI -0.07 to 0.15 ), retinopathy of prematurity (one study; RR 2.27 , $95 \%$ CI 0.85 to 6.11 ; RD $0.25,95 \% \mathrm{CI}-0.02$ to 0.53 ), and severe retinopathy of prematurity (one study; RR $2.73,95 \%$ CI 0.31 to $24.14 ; \mathrm{RD} 0.09,95 \%$ CI -0.09 to 0.26 ). None of the included trials reported on the other specified outcomes of this review (i.e., cerebellar hemorrhage, brain magnetic resonance imaging abnormalities, impairment in cerebral hemodynamics, long-term neurodevelopmental outcomes, and major neurodevelopmental disability). The quality of evidence supporting these findings is limited owing to the imprecision of the estimates. We identified no ongoing studies.

\section{Authors' conclusions}

Given the imprecision of the estimate, results of this systematic review are consistent with beneficial or detrimental effects of a supine head midline position versus a lateral position and do not provide a definitive answer to the review question.

\section{PLAIN LANGUAGESUMMARY}

\section{Head midline (central) position for preventing intraventricular hemorrhage (i.e., bleeding in the brain) in very preterm infants}

Review question: Does head midline position reduce the risk of intraventricular hemorrhage (i.e., bleeding in the brain) and mortality in very preterm infants?

Background: Intraventricular hemorrhage (i.e., bleeding in the brain) occurs in $25 \%$ of very low birth weight infants and may be caused by multiple factors. Head position may affect how the blood circulates within the brain and thus may be involved in development of intraventricular hemorrhage. Turning the head toward one side may limit return of blood in the veins of the same side and may increase pressure and the amount of blood within the brain. It has been suggested that this might be avoided if the patient is in supine (lying on the back) midline (central) position, especially during the first two to three days of life, when risk of intraventricular hemorrhage is greatest.

Study characteristics: We included two studies comparing supine midline head position versus supine head rotated $90^{\circ}$.

Key results: This review of trials found too little evidence to show positive or negative effects of supine (lying on the back) midline head position for prevention of intraventricular hemorrhage (i.e., bleeding within the brain), mortality, or any other relevant outcomes

Head midline position for preventing the occurrence or extension of germinal matrix-intraventricular hemorrhage in preterm infants 
in very preterm neonates. More research is needed. We found no trials that compared supine (lying on the back) versus prone (lying on the stomach) midline head position, and no trials that compared effects of head tilting (elevating the head of the incubator upward).

Conclusions: Results of this systematic review are consistent with beneficial or detrimental effects of a supine head midline position and do not provide a definitive answer to the review question. 


\section{S U M M A Y OF F INDINGS F OR THE MAIN COMPARISON [Explanation]}

Supine midline head position with the bed at $0^{\circ}$ versus supine head rotated $90^{\circ}$ with the bed at $0^{\circ}$ for preventing occurrence or extension of germinal matrix-intraventricular hemorrhage in preterm infants

Patient or population: very preterm infants (i.e., $\leq 32$ weeks' gestational age) of any birth weight

Setting: neonatal intensive care units (included trials conducted in Saudi Arabia)

Intervention: supine midline head position with the bed at $0^{\circ}$

Comparison: supine head rotated $90^{\circ}$ with the bed at $0^{\circ}$

\begin{tabular}{ll} 
Outcomes & Illustrative comparative risks* $(95 \% \mathrm{Cl})$ \\
\hline Assumed risk & Corresponding risk \\
\hline Control & $\begin{array}{l}\text { Supine midline head } \\
\text { position with the bed at } \\
0^{\circ} \text { vs supine head ro- } \\
\text { tated } 90^{\circ} \text { with the bed } \\
\text { at } 0^{\circ}\end{array}$ \\
\hline
\end{tabular}

Intraventricular hem- High-risk population

orrhage, any grade

\section{Relative effect}

$(95 \% \mathrm{Cl})$
No. of participants

(studies)
Quality of the evidence Comments (GRADE)

\section{RR 1.14}

(0.55 to 2.35)
110

(2 studies)

\section{$\oplus \oplus \bigcirc \bigcirc$}

low
- High risk for performance bias in both included trials, although blinding for outcome assessors was provided; unclear risk for selection and reporting bias in Al-Abdi 2015 and Al-Abdi 2011,

respectively. Downgraded by one level

- Precision: lack of precision due to smal sample size and wide 


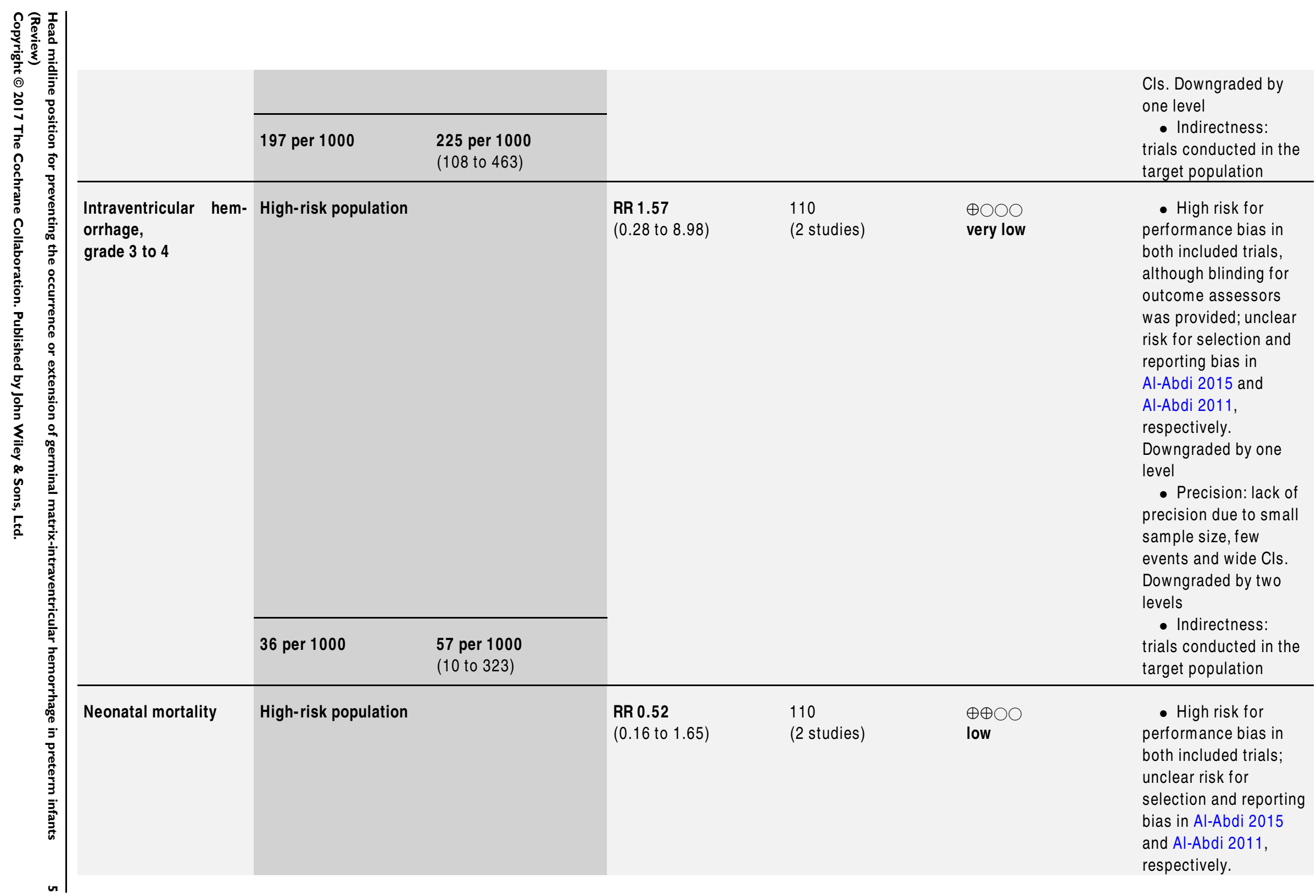


Downgraded by one level

- Precision: lack of precision due to small sample size and wide Cls. Downgraded by one level

- Indirectness: trials conducted in the target population

*The basis for the assumed risk (e.g., median control group risk across studies) is provided in footnotes. The corresponding risk (and its $95 \%$ confidence interval) is based on assumed risk in the comparison group and the relative effect of the intervention (and its $95 \% \mathrm{Cl}$ )

Cl: confidence interval; RR: risk ratio

GRADE Working Group grades of evidence

High quality: Further research is very unlikely to change our confidence in the estimate of effect

Moderate quality: Further research is likely to have an important impact on our confidence in the estimate of effect and may change the estimate

Low quality: Further research is very likely to have an important impact on our confidence in the estimate of effect and is likely to change the estimate

Very low quality: We are very uncertain about the estimate

None of the included trials reported on the other outcomes that we planned to present in this table (i.e., cerebellar hemorrhage on brain ultrasound; retinopathy of prematurity; long-term neurodevelopmental outcome; and major neurodevelopmental disability). 


\section{B A C K G RO U N D}

\section{Description of the condition}

Preterm birth remains a major risk factor for development of germinal matrix-intraventricular hemorrhage (GM-IVH), which occurs in $25 \%$ of very low birth weight (VLBW) infants (Canadian Neonatal Network 2014; Vermont Oxford Network 2013). Often, these hemorrhages occur during the first days of life (Dolfin 1983). Complications of GM-IVH, including periventricular hemorrhagic infarction (PVHI), posthemorrhagic ventricular dilatation (PHVD), and associated cerebellar hemorrhagic injury (CHI) and periventricular leukomalacia (PVL), are critical determinants of neonatal morbidity, mortality, and long-term neurodevelopmental sequelae (Sherlock 2005). Although modern perinatal medicine has led to a significant decrease in the overall incidence of GMIVH among preterm infants (i.e., from $50 \%$ in the late 1970 s to current rates of $15 \%$ to $25 \%$ ) (Hamrick 2004; Horbar 2002; Philip 1989), GM-IVH continues to present a significant problem in the modern neonatal intensive care unit. The origin of GM-IVH is multifactorial, complex, and heterogeneous. Inherent fragility of the germinal matrix vasculature sets the ground for hemorrhage, and fluctuation in cerebral blood flow induces rupture of the vasculature. Furthermore, the germinal matrix lies within an arterial end zone, and it is directly connected to the deep galenic venous system (Nakamura 1990; Pape 1979), thereby exposing it to insults of arterial ischemia-reperfusion and venous congestion (Pape 1979; Takashima 1978). The immature deep galenic system is prone to venous congestion and stasis, making it of potentially major importance for development of GM-IVH and its complications (Pape 1979; Volpe 2008). It is unknown what proportion of GM-IVH might occur because of this phenomenon. Nevertheless, many institutions adopt the practice of head midline position. Vaginal delivery, low Apgar score, severe respiratory distress syndrome, pneumothorax, hypoxia, hypercapnia, seizures, patent ductus arteriosus, infection, and other factors seem to primarily increase fluctuations in cerebral blood flow, thus representing important risk factors for development of GM-IVH (Ballabh 2014).

\section{Description of the intervention}

It has been suggested that head position may affect cerebral hemodynamics in the preterm newborn and might be involved indirectly in development of GM-IVH. Doppler studies in term infants have shown that turning the head toward one side functionally occluded jugular venous drainage on the ipsilateral side (Cowan 1985). Moreover, an increase in intracranial pressure (Cowan 1985; Emery 1983) and in cerebral blood volume (CBV) (Pellicer 2002) after head rotation, caused by obstruction of the homolateral jugular veins, has been reported. Thus, it has been suggested that cerebral venous pressure is reduced and hydrostatic brain drainage improved if the patient is in supine midline position with the bed tilted $30^{\circ}$ (Cowan 1985; Emery 1983). Researchers have reported an increase in cerebral blood flow (CBF) in the supine position and an increase in partial pressure of oxygen $\left(\mathrm{PO}_{2}\right)$ in the prone position in stable preterm infants (Bembich 2012). However, Ancora's study results did not show significant changes in the tissue hemoglobin index (which is proportionate to changes in $\mathrm{CBV}$ ) nor in oxygenation. Only infants with low gestational age ( $<26$ weeks) showed a reduction in CBV with head rotation (Ancora 2010). In addition, ventilatory support has been shown to influence brain hemodynamics (Cowan 1987): Newborns on mechanical ventilation showed an increase in CBV during inspiration compared with those breathing spontaneously (Leahy 1982). However, nasal continuous positive airway pressure (nCPAP) did not seem to have an effect on CBV nor on CBF among preterm infants (Dani 2007; Moritz 2008).

The definition of head midline position is complex, as the position of the body may have a relevant impact. In the supine position, the infant's head is maintained in alignment with the midline. In the prone position, the head has to be turned to the side, so the head midline position is not feasible. In the lateral position, the midline position might be achieved if the head is kept aligned with midline. Maintenance of this position may require the presence of physical aids, such as nests or pillows, and active surveillance by nurses. It has been reported that the midline position in the lateral decubitus during kangaroo care might be associated with improved early neuromotor development as assessed by the Dubowitz score (Barradas 2006). Midline position should be kept at least when the incidence of GM-IVH is greatest, that is, during the first two to three days of life. It is unknown, however, if strict observance of the midline position might confer any disadvantages, and if the head midline position with the infant supine is different from the head midline position with the infant lying on the side.

\section{How the intervention might work}

An intubated preterm infant's head may be turned toward one side (facing the ventilator, e.g., a high-frequency oscillator) for prolonged periods. As impaired venous drainage and decreased cerebral tissue oxygenation are factors implicated in the pathogenesis of IVH (Noori 2014; Osborn 2003; Takashima 2009), midline head positioning during the early transitional period has been included in recent IVH prevention bundles at many institutions, albeit without strong data to support the practice (McLendon 2003; Nankervis 2010; Obladen 2008). Midline head positioning during the early transitional period might prevent the occurrence of IVH through improved venous drainage and cerebral oxygenation. 


\section{Why it is important to do this review}

As noted above, GM-IVH occurs in 25\% of VLBW infants (Canadian Neonatal Network 2014; Vermont Oxford Network 2013). Midline head positioning has been included in recent GM-IVH prevention bundles at many institutions, albeit without strong data to support the practice (McLendon 2003; Nankervis 2010; Obladen 2008). One review recommended midline head position for preterm infants on the basis of physiological data and expert opinion; however, review authors identified no controlled trials for inclusion (Malusky 2011). Moreover, midline positioning with bed elevation of $30^{\circ}$ has been identified as a "potentially better practice" for prevention of GM-IVH, although review authors rated the quality of evidence as low (Carteaux 2003). This systematic review will help clinicians and policy makers to provide specific recommendations about optimal head positioning, with an important impact on neonatal health and long-term outcomes for the very preterm infant.

\section{O B J E C T I VES}

\section{Primary objective}

To assess whether head midline position is more effective than any other head position for preventing or extending germinal matrixintraventricular hemorrhage in infants born at $\leq 32$ weeks' gestational age.

\section{Secondary objectives}

To perform subgroup analyses regarding gestational age, birth weight, intubated versus not intubated, and with or without GMIVH at trial entry (see Subgroup analysis and investigation of heterogeneity).

\section{METHODS}

\section{Criteria for considering studies for this review}

\section{Types of studies}

We included prospective randomized clinical controlled trials, quasi-randomized trials, and cluster-randomized controlled trials. We excluded cross-over trials because the intervention might have a lasting effect that compromises entry to subsequent periods of the trial.

\section{Types of participants}

We included very preterm infants (i.e., $\leq 32$ weeks' gestational age) of any birth weight, admitted to neonatal intensive care units. We included studies enrolling infants with unknown GM-IVH status at enrollment; if known, we planned to perform subgroup analysis on the presence of GM-IVH at study entry.

We planned to include studies enrolling infants with existing GM$\mathrm{IVH}$ and to assess extension of hemorrhage in a subgroup of infants.

\section{Types of interventions}

Placing newborns in a head midline position compared with placing them in a prone or lateral decubitus position, or undertaking a strategy of regular position change, or having no prespecified position.

We planned to analyze horizontal (flat) versus head elevated positions separately for all body positions.

We planned to conduct the following comparisons.

- Supine midline head position versus any other supine head position

- Supine midline head position with the bed at $0^{\circ}$ versus supine head rotated $90^{\circ}$ with the bed at $0^{\circ}$

- Supine midline head position with the bed at $0^{\circ}$ versus supine head rotated $90^{\circ}$ with the bed tilted $\geq 30^{\circ}$

- Supine midline head position with the bed tilted $\geq$ $30^{\circ}$ versus supine head rotated $90^{\circ}$ with the bed at $0^{\circ}$

- Supine midline head position with the bed tilted $\geq$ $30^{\circ}$ versus supine head rotated $90^{\circ}$ with the bed tilted $\geq 30^{\circ}$

- Supine midline head position versus any other prone head position

- Supine midline head position with the bed at $0^{\circ}$ versus prone head rotated $90^{\circ}$ with the bed at $0^{\circ}$

- Supine midline head position with the bed at $0^{\circ}$ versus prone head rotated $90^{\circ}$ with the bed tilted $\geq 30^{\circ}$

- Supine midline head position with the bed tilted $\geq$ $30^{\circ}$ versus prone head rotated $90^{\circ}$ with the bed at $0^{\circ}$

- Supine midline head position with the bed tilted $\geq$ $30^{\circ}$ versus prone head rotated $90^{\circ}$ with the bed tilted $\geq 30^{\circ}$

- Supine midline head position with the bed at $0^{\circ}$ versus supine midline head position with the bed tilted $\geq 30^{\circ}$

As the aim of this review is to assess the ability of head position to prevent GM-IVH, we included trials in which the intervention is started within the first 48 hours of life.

\section{Types of outcome measures}

\section{Primary outcomes}

- Any germinal matrix-intraventricular hemorrhage: any IVH, grades 1 to 4 (according to Papile classification [Papile 1978])

Head midline position for preventing the occurrence or extension of germinal matrix-intraventricular hemorrhage in preterm infants 
- Severe IVH: ultrasound diagnosis grades 3 and 4 (according to Papile classification [Papile 1978])

- Neonatal death (first 28 days or during initial hospitalization)

\section{Secondary outcomes}

- Cerebellar hemorrhage on brain ultrasound in the first month of life (yes/no; Graça 2013)

- Cystic periventricular leukomalacia on brain ultrasound in the first month of life

- Brain magnetic resonance imaging (MRI) abnormalities at term equivalent age (yes/no), defined as white matter lesions (i.e., cavitations; Rutherford 2010) and punctate lesions (Cornette 2002); GM-IVH (Parodi 2015); or cerebellar hemorrhage (Fumagalli 2009; Limperopoulos 2007)

- Impairment in cerebral hemodynamics during the first three days of life, assessed on cerebral near-infrared spectroscopy (NIRS)

- Retinopathy of prematurity: any and severe ( $\geq$ stage 3; ICROP 1984)

- Long-term neurodevelopmental outcomes (yes/no): cerebral palsy on physician assessment, developmental delay (i.e., IQ 2 standard deviations [SDs] below the mean on validated assessment tools such as Bayley Mental Developmental Index) (Bayley 1993; Bayley 2006)

- Major neurodevelopmental disability: cerebral palsy, developmental delay (Bayley Mental Developmental Index [Bayley 1993; Bayley 2006] or Griffiths Mental Development Scale [Griffiths 1954] assessment > 2 SDs below the mean), intellectual impairment (IQ $>2$ SDs below the mean), blindness (vision $<6 / 60$ in both eyes), or sensorineural deafness requiring amplification (Jacobs 2013). We planned to evaluate each of these components as a separate outcome and to extract data on each long-term outcome from studies that evaluated children after 18 months' chronological age. We planned to separately assess data on children 18 to 24 months of age and on those 3 to 5 years of age

\section{Search methods for identification of studies}

\section{Electronic searches}

We used criteria and standard methods of Cochrane and the Cochrane Neonatal Review Group (see the Cochrane Neonatal Group search strategy for specialized register).

We conducted a comprehensive search that included the Cochrane Central Register of Controlled Trials (CENTRAL; 2016, Issue 8) in the Cochrane Library; MEDLINE via PubMed (1966 to September 16, 2016); Embase (1980 to September 16, 2016); and the Cumulative Index to Nursing and Allied Health Literature
(CINAHL; 1982 to September 16, 2016), using the following search terms: (head midline OR prone OR lateral decubitus OR posture OR lateral alternant OR ((head OR body) AND (position OR positioning))), plus database-specific limiters for randomized controlled trials (RCTs) and neonates (see Appendix 1 for full search strategies for each database). We did not apply language restrictions.

\section{Searching other resources}

We searched clinical trials registries for ongoing and recently completed trials (clinicaltrials.gov; the World Health Organization International Trials Registry and Platform [www.whoint/ictrp/ search/en/], the ISRCTN Registry).

\section{Data collection and analysis}

We used standard methods of the Cochrane Neonatal Review Group, as described below.

\section{Selection of studies}

Two review authors (OR, MB) independently searched for and identified eligible trials that met the inclusion criteria of this review. We screened titles and abstracts to identify potentially relevant citations, and retrieved the full texts of all potentially relevant articles; we independently assessed the eligibility of studies by filling out eligibility forms designed in accordance with the specified inclusion criteria. We reviewed studies for relevance by assessing study design, types of participants, interventions provided, and outcome measures reported. We resolved disagreements by discussion and, if necessary, by consultation with a third review author (MGC). We provided in the Characteristics of excluded studies table details of studies excluded from the review, along with reasons for exclusion. We contacted trial authors if details of primary trials were not clear.

\section{Data extraction and management}

Two review authors (OR, MB) independently extracted data using a data extraction form developed ad hoc and integrated with a modified version of the Cochrane Effective Practice and Organisation of Care Group data collection checklist (Cochrane EPOC Group 2015)

We extracted the following characteristics from each included study.

- Administrative details: study author(s); published or unpublished; year of publication; year in which study was conducted; details of other relevant papers cited.

- Details of the study: study design; type, duration, and completeness of follow-up (e.g., > 80\%); country and location of study; informed consent; ethics approval. 
- Details of participants: sex, birth weight, gestational age, number of participants.

- Details of interventions: initiation and duration of head midline position; co-intervention with horizontal versus head elevated position; use of physical aids to maintain head position.

- Details of outcomes as mentioned above under Types of outcome measures.

We resolved disagreements by discussion. We planned to describe ongoing studies identified by our search, when available, detailing the primary author, research question(s), methods, and outcome measures, together with an estimate of the reporting date.

When any queries arose, or when we required additional data, we contacted trial investigators/authors for clarification.

Two review authors (MGC, MB) used Cochrane statistical software (RevMan 2014) for data entry.

\section{Assessment of risk of bias in included studies}

Two review authors (OR, MGC) independently assessed risk of bias in all included studies using the Cochrane tool designed to assess risk of bias (Higgins 2011).

We assessed the following items.

- Random sequence generation: selection bias due to inadequate generation of a randomized sequence.

- Allocation concealment: selection bias due to inadequate concealment of allocations before assignment.

- Blinding of participants and personnel: performance bias due to knowledge of allocated interventions by participants and personnel during the study.

- Blinding of outcome assessment: detection bias due to knowledge of allocated interventions by outcome assessors.

- Incomplete outcome data: attrition bias due to quantity, nature, or handling of incomplete outcome data.

- Selective reporting: reporting bias due to selective outcome reporting.

- Other bias: bias due to problems not covered elsewhere in the table.

We used a "Risk of bias" graph to illustrate risk across studies. We planned to resolve disagreements by consensus and, if necessary, by consultation with a third review author (MB).

\section{Random sequence generation and allocation concealment (selection bias)}

\section{Random sequence generation}

For each included study, we categorized as follows the risk of bias regarding random sequence generation.

- Low risk: Investigators describe a random component in the sequence generation process such as referring to a random number table, using a computer random number generator, tossing a coin, shuffling cards or envelopes, throwing dice, drawing lots, and minimizing.

- High risk: Investigators describe a nonrandom component in the sequence generation process (sequence generated by odd or even date of birth, sequence generated by some rule based on date or day of admission, sequence generated by some rule based on hospital or clinic record number, allocation by judgment of the clinician, allocation by preference of the participant, allocation based on results of a laboratory test or a series of tests, allocation by availability of the intervention).

- Unclear risk: No or unclear information is provided.

\section{Allocation concealment}

For each included study, we categorized as follows the risk of bias regarding allocation concealment.

- Low risk: Participants and investigators enrolling participants could not foresee assignment because one of the following, or an equivalent method, was used to conceal allocation: central allocation (including telephone, web-based, and pharmacy-controlled randomization), sequentially numbered drug containers or those of identical appearance, sequentially numbered sealed opaque envelopes.

- High risk: Participants and investigators enrolling participants could possibly foresee assignments and thus introduce selection bias, such as allocation based on open random allocation schedule (e.g., a list of random numbers), unsealed or non opaque envelopes, alternation or rotation, date of birth, case record number.

- Unclear risk: No or unclear information is provided.

\section{Blinding of study participants and personnel (performance bias)}

Care providers cannot be blinded to the intervention.

\section{Blinding of outcome assessors (detection bias)}

For each included study, we categorized as follows the methods used to blind outcome assessors from knowledge of which intervention a participant received.

- Criteria for a judgment of "low risk" of bias: No blinding or incomplete blinding is described, but review authors judge that the outcome is not likely to be influenced by lack of blinding; blinding of participants and key study personnel is ensured, and it is unlikely that blinding could have been broken.

- Criteria for a judgment of "high risk" of bias: No blinding of outcome assessment is described, but review authors judge that the outcome measurement is not likely to be influenced by lack of blinding; blinding of outcome assessment is described, but it is likely that blinding could have been broken, and the outcome measurement is likely to be influenced by lack of blinding.

- Unclear risk: No or unclear information is provided. 


\section{Incomplete outcome data (attrition bias)}

For each included study and for each outcome, we described the completeness of data including attrition and exclusions from the analysis as follows.

- Criteria for a judgment of "low risk" of bias include:

- no missing outcome data;

- reasons for missing outcome data unlikely to be

related to true outcome (for survival data, censoring unlikely to introduce bias);

- missing outcome data balanced in numbers across intervention groups, with similar reasons for missing data across groups;

o for dichotomous outcome data, proportion of missing outcomes compared with observed event risk not enough to have a clinically relevant impact on the intervention effect estimate;

- for continuous outcome data, plausible effect size (difference in means or standardized difference in means) among missing outcomes not enough to have a clinically relevant impact on observed effect size; and

- missing data imputed by appropriate methods.

- Criteria for a judgment of "high risk" of bias include:

$\circ$ reasons for missing outcome data likely to be related to true outcome, with imbalance in numbers or reasons for missing data across intervention groups;

- for dichotomous outcome data, proportion of missing outcomes compared with observed event risk enough to induce clinically relevant bias in the intervention effect estimate;

o for continuous outcome data, plausible effect size (difference in means or standardized difference in means) among missing outcomes enough to induce clinically relevant bias in observed effect size;

○ "as-treated" analysis done with substantial departure of the intervention received from that assigned at randomization; and

$\circ$ potentially inappropriate application of simple imputation.

- Unclear risk: No or unclear information is provided.

\section{Selective reporting (reporting bias)}

For each included study, we described how we investigated the risk of selective outcome reporting bias and what we found. We attempted to access all protocols of included studies through clinical trials registries (e.g., ClinicalTrials.gov [https://clinicaltrials.gov/], the International Standard Randomised Controlled Trial Number [ISRCTN] registry [http://www.controlled-trials.com/]) and by direct contact with study authors.

We assessed study methods as follows.

- Low risk: The study protocol is available, and all of the study's prespecified (primary and secondary) outcomes of interest in the review have been reported in the prespecified way; the study protocol is not available, but it is clear that published reports include all expected outcomes, including those that were prespecified (convincing text of this nature may be uncommon).

- High risk: Not all of the study's prespecified primary outcomes have been reported; one or more primary outcomes were reported using measurements, analysis methods, or subsets of data (e.g., subscales) that were not prespecified; one or more reported primary outcomes were not prespecified (unless clear justification for their reporting is provided, such as an unexpected adverse effect); one or more outcomes of interest in the review were reported incompletely, so that they cannot be entered into a meta-analysis; the study report fails to include results for a key outcome that would be expected to have been reported for such a study.

- Unclear risk: No or unclear information is provided (the study protocol is not available).

\section{Other potential sources of bias (other bias)}

For each included study, we described any important concerns that we had about other possible sources of bias (e.g., whether a potential source of bias was related to the specific study design used).

We assessed whether each study was free of other problems that could put it at risk of bias as follows.

- Low risk: The study appears to be free of other sources of bias.

- High risk: The study has at least one important risk of bias (e.g., the study had a potential source of bias related to the specific study design used, was claimed to have been fraudulent, had some other problem).

- Unclear risk: Risk of bias may be present, but information is insufficient to assess whether an important risk of bias exists, or the rationale or evidence that an identified problem will introduce bias is insufficient.

\section{Measures of treatment effect}

We followed standard methods of the Cochrane Neonatal Review Group for data synthesis. We extracted categorical data for each intervention group and calculated risk ratios (RRs) and absolute risk differences (RDs). We planned to obtain means and standard deviations for continuous data and to perform analyses using mean differences (MDs). For each measure of effect, we calculated corresponding $95 \%$ confidence intervals (CIs). We planned to present the number needed to treat for an additional beneficial outcome and the number needed to treat for an additional harmful outcome (NNTB/NNTH) when we found RDs to be statistically significant $(\mathrm{P}<0.05)$.

\section{Unit of analysis issues}

The unit of randomization was the intended unit of analysis (individual neonate). If we found any cluster-randomized controlled 
trials, we planned to adjust analysis for the designed effect using methods stated in the Cochrane Handbook for Systematic Reviews of Interventions (Higgins 2011).

\section{Dealing with missing data}

We planned to obtain a dropout rate for each study. We planned to consider a dropout rate $>20 \%$ as significant. If we found a significant dropout rate, we planned to contact study author(s) to request additional data. We planned to perform a sensitivity analysis to evaluate overall results with and without inclusion of studies with a significant dropout rate. If a study reported outcomes only for participants completing the trial or only for participants who followed the protocol, we planned to contact study author(s) to ask them to provide additional information to facilitate an intention-to-treat analysis; in instances when this was not possible, we planned to perform a complete case analysis.

\section{Assessment of heterogeneity}

We planned to assess clinical heterogeneity by comparing the distribution of important participant factors between trials and trial factors (randomization concealment, blinding of outcome assessment, loss to follow-up, treatment type, co-interventions). We planned to assess statistical heterogeneity by examining the $\mathrm{I}^{2}$ statistic (Higgins 2011) - a quantity that describes the proportion of variation in point estimates that is due to variability across studies rather than to sampling error.

We planned to interpret the $\mathrm{I}^{2}$ statistic as follows, as described by Higgins 2003.

- $<25 \%$ : no (none) heterogeneity.

- $25 \%$ to $49 \%$ : low heterogeneity.

- $50 \%$ to $74 \%$ : moderate heterogeneity.

- $\geq 75 \%$ : high heterogeneity.

We planned to consider statistical heterogeneity substantial when $\mathrm{I}^{2} \geq 50 \%$. In addition, we planned to employ the $\mathrm{Chi}^{2}$ test of homogeneity to determine the strength of evidence that heterogeneity is genuine. We planned to explore clinical variation across studies by comparing the distribution of important participant factors among trials and trial factors (randomization concealment, blinding of outcome assessment, loss to follow-up, treatment types, and co-interventions). We planned to consider a threshold of $\mathrm{P}<0.1$ as an indicator of whether heterogeneity (genuine variation in effect sizes) was present.

\section{Assessment of reporting biases}

We planned to investigate publication by using funnel plots if we included 10 or more clinical trials in the systematic review (Egger 1997; Higgins 2011).

\section{Data synthesis}

We summarized all eligible studies in RevMan 5.3 (RevMan 2014). We used standard methods of the Cochrane Neonatal Review Group to synthesize data using typical RR, RD, NNTB, NNTH, $\mathrm{MD}$, and $95 \%$ CIs if we included more than one trial in the metaanalysis. We performed a meta-analysis of data from included trials by using a fixed-effect model.

\section{Quality of evidence}

We used the Grading of Recommendations, Assessment, Development, and Evaluation (GRADE) approach, as outlined in The GRADE Handbook (Schünemann 2013), to assess the quality of evidence for the following (clinically relevant) outcomes: any intraventricular hemorrhage; severe intraventricular hemorrhage; death during initial hospitalization; cerebellar hemorrhage on brain ultrasound; retinopathy of prematurity; long-term neurodevelopmental outcome; and major neurodevelopmental disability.

Two review authors independently assessed the quality of the evidence for each of the outcomes above. We considered evidence from randomized controlled trials as high quality but downgraded the evidence one level for serious (and two levels for very serious) limitations on the basis of the following: design (risk of bias), consistency across studies, directness of evidence, precision of estimates, and presence of publication bias. We used the GRADEpro GDT Guideline Development Tool to create a "Summary of findings" table to report the quality of the evidence.

The GRADE approach yields an assessment of the quality of a body of evidence according to one of four grades.

- High: We are very confident that the true effect lies close to that of the estimate of effect.

- Moderate: We are moderately confident in the effect estimate: The true effect is likely to be close to the estimate of effect but may be substantially different.

- Low: Our confidence in the effect estimate is limited: The true effect may be substantially different from the estimate of effect.

- Very low: We have very little confidence in the effect estimate: The true effect is likely to be substantially different from the estimate of effect.

\section{Subgroup analysis and investigation of heterogeneity}

We planned to present data from the following subgroups.

- Gestational age (with two subgroups, $<26$ weeks vs $\geq 26$ weeks).

- Birth weight (with two subgroups, $<1000$ grams vs $\geq 1000$ grams).

- Intubated versus not intubated.

- With or without GM-IVH (any grade) at trial entry.

Head midline position for preventing the occurrence or extension of germinal matrix-intraventricular hemorrhage in preterm infants 


\section{Sensitivity analysis}

We planned to conduct sensitivity analyses to explore effects of the methodological quality of trials, checking to ascertain whether studies with high risk of bias will overestimate the effects of treatment.

\section{RE S U L T S}

\section{Description of studies}

See Characteristics of included studies, Characteristics of excluded studies, and Characteristics of studies awaiting classification.

\section{Results of the search}

The literature search run in September 2016 identified 2696 references (see Figure 1). After screening, we assessed five full-text articles for eligibility. We excluded two trials (Antunes 2003; Wu 2015) and considered for inclusion three RCTs (Al-Abdi 2011; Al-Abdi 2015; Jalali 2012). However, we included only two studies in the qualitative and quantitative synthesis (Al-Abdi 2011; Al-Abdi 2015). We classified the third study in the list of Studies awaiting classification because approximately half of the included infants had a gestational age greater than the inclusion criterion of this review; moreover, study authors reported no outcomes specified in this review (Jalali 2012). 
Figure I. Study flow diagram.

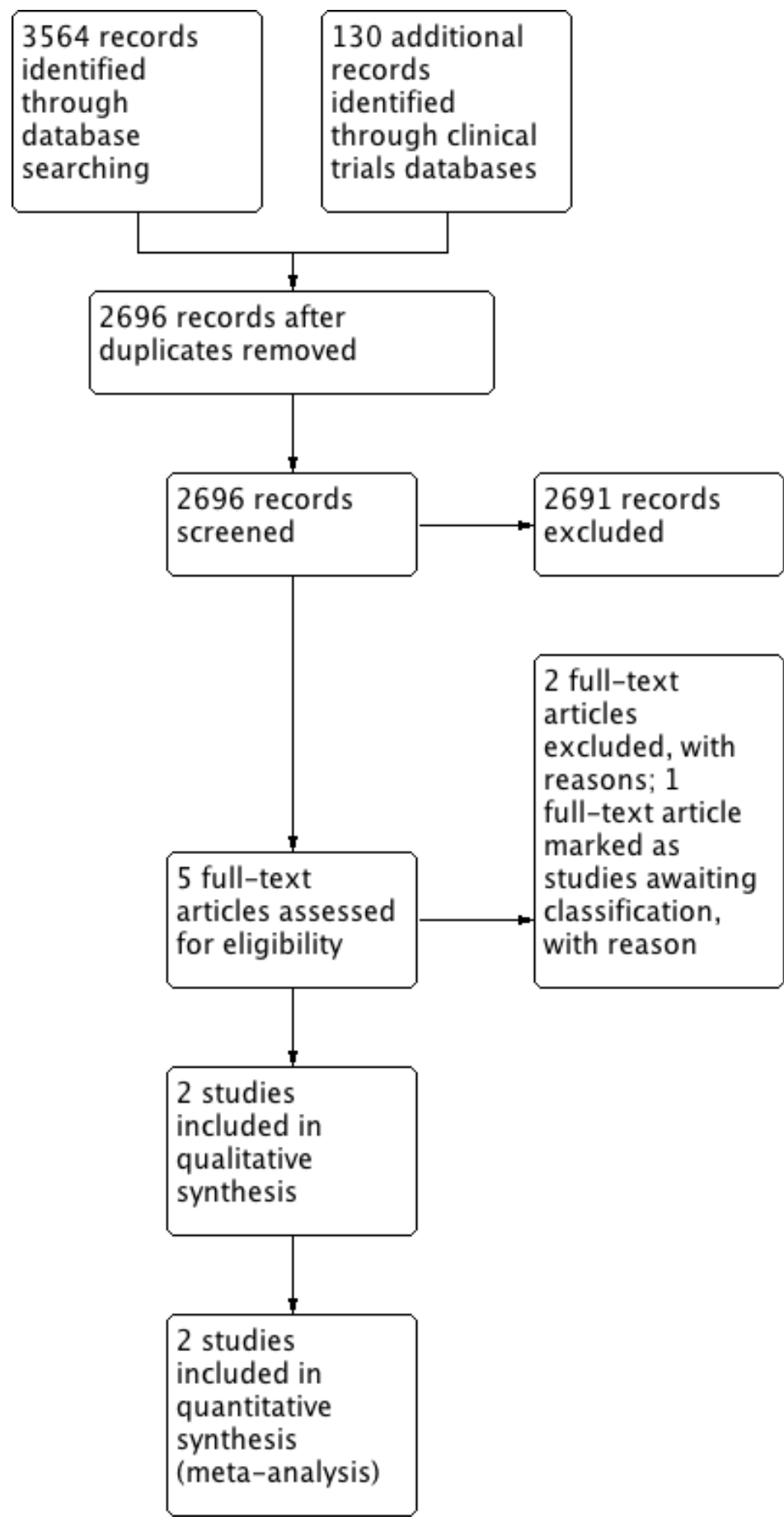


We did not find other relevant studies by searching clinical trial registries.

\section{Included studies}

Two RCTs recruiting 110 infants met the inclusion criteria (AlAbdi 2011; Al-Abdi 2015). We have listed the details of these trials in the Characteristics of included studies section. Both trials compared supine midline head position (with the bed at $0^{\circ}$ ) versus supine head rotated $90^{\circ}$ (with the bed at $0^{\circ}$ ). We found no trials that compared supine versus prone midline head position, and no trials that compared effects of head tilting.

Al-Abdi 2011 was a randomized parallel clinical trial conducted at King Abdulaziz Hospital, Al-Ahsa, Saudi Arabia, including 48 preterm infants in the period 2008-2009. Preterm infants were enrolled in the trial if they met the following criteria: inborn; gestational age $<30$ weeks; and postnatal age $<2$ hours. Exclusion criteria included presence of lethal congenital anomalies, hypoxic ischemic encephalopathy, and need for full cardiopulmonary resuscitation at birth. Infants lying on beds at $0^{\circ}$ were randomly assigned to be cared for in a supine midline or supine lateral head position. In the supine midline position, the infant's chin was kept at a $90^{\circ}$ angle to the bed. In the lateral head position, the head was tilted $90^{\circ}$ to either side. At enrollment, it was left to the bedside nurse to place the head in the right-tilted or left-titled position. Since then, infants' heads were kept in their primary assigned positions throughout the first week of life. Correctness of infants' head positions was checked every 6 hours by the bedside nurse. Randomization was stratified on the basis of gender and gestational age ( $<27$ or 27 to $29+6$ weeks). The primary outcome was rate of intraventricular hemorrhage of all grades. Two radiologists who were blinded to head position assignments independently reported laterality and grade of IVH according to Papile's criteria (Papile 1978). If discrepancy between the two radiologists was apparent, we used the report of a third radiologist. Timing of head ultrasound examinations was based on our established IVH screening guidelines: (1) at 5 to 7 days of life for stable preterm infants $\leq 32$ weeks' gestational age (GA); (2) subsequent head ultrasound follow-up examinations at 14 and 28 days of life, or before discharge; (3) head ultrasound examination as soon as clinical suspicion of IVH was raised; (4) if IVH was detected, a second head ultrasound examination repeated 5 to 7 days later; and (5) before commencement of indomethacin for treatment of patent ductus arteriosus. Investigators noted no statistically significant differences in the baseline characteristics of studied infants. Twenty-three infants were cared for in the midline head position and 25 in the lateral head position. One infant in each group had no head ultrasound owing to early death, and both infants were included in the analysis. Among infants in the head midline group, 12 were cared for in a left-tilted and 13 in a right-tilted midline head position. During the first week of life, the incidence of IVH in the midline head position was $26 \%(6 / 23)$ versus $20 \%(5 / 25)$ in the lateral head position (risk ratio $[\mathrm{RR}] 1.30 ; 95 \%$ confidence interval $[\mathrm{CI}] 0.46$ to 3.70; $\mathrm{P}=0.62$ ). Among infants who developed IVH, 4 in the midline and 3 in the lateral head position had normal first head ultrasound carried out on the second day of life before indomethacin for patent ductus arteriosus treatment. Grade 3 to 4 IVH developed in $2(9 \%)$ infants in the midline head position versus $1(4 \%)$ infant in the lateral head position (RR 1.4, 95\% CI 0.61 to 3.37 ; $\mathrm{P}=0.94)$. Bilateral IVH developed in $3(13 \%)$ in the midline head position versus $2(8 \%)$ in the lateral head position (RR 1.6, 95\% CI 0.30 to $8.90 ; \mathrm{P}=0.92$ ). Secondary analysis showed that the incidence of IVH in left-tilted lateral head position was $25 \%$ $(3 / 12)$ versus $15 \%(2 / 13)$ in right-tilted lateral head position (RR $1.63,95 \%$ CI 0.33 to $8.11 ; \mathrm{P}=0.92$ ). In addition, we obtained data for the following outcomes directly from trial authors: death - one infant in the midline group and 5 in the lateral group (all deaths occurred within the first 2 weeks of life); retinopathy of prematurity, any stage - 10 infants in the midline group and 4 in the lateral head position group; retinopathy of prematurity, severe ( $\geq$ stage 3 ) - 3 infants in the midline group and 1 in the lateral head position group; cystic periventricular leukomalacia - 1 infant in the midline group and 0 in the lateral head position group. No data on long-term follow-up were available.

Al-Abdi 2015 was a multicenter (Saudi Arabia) randomized parallel clinical trial that included 62 preterm neonates $(<30$ weeks' gestation) within 2 hours of life and without IVH at 12 hours of life. Exclusion criteria included the following: outborn; lethal congenital anomalies; hypoxic ischemic encephalopathy; and external cardiac compression or epinephrine administration at birth. Head ultrasound scan was performed within 12 hours of life, at 7 days of life, and at the physician's discretion. Head ultrasound scan was reported by three radiologists who were blinded to assignment of head position. Infants lying on $0^{\circ}$ beds were randomly assigned to be cared for in a supine midline $(\mathrm{n}=31)$ or supine lateral head $(\mathrm{n}=31)$ position throughout the first week of life. Severity score of IVH was calculated according to an IVH severity score defined by one of the study authors (Al Abdi's score). The planned sample size was 600 (alpha 5\%; power $80 \%$ ). However, the study was prematurely terminated when only 71 neonates $(12 \%)$ had been recruited owing to low accrual rate. The trial was registered on clinicaltrials.gov, and findings were presented at the Pediatric Academic Societies (PAS) in 2015. Risk of IVH was exactly the same in both groups (6/31 [19.4\%]; RR 1.0, 95\% CI 0.36 to 2.76 ; P > 0.99). Risk ratio of left-sided IVH in flat midline versus flat right lateral groups was 2.0 (95\% CI 0.94 to 10.13 ; P = 0.39). Risk ratio of right-sided IVH groups was 1.0 (95\% CI 0.32 to 3.11 ; $\mathrm{P}=0.39)$. Risk ratio of bilateral IVH in flat midline versus flat right lateral was 3.0 (95\% CI 0.33 to 27.29 ; $\mathrm{P}=0.31)$. Median of 
IVH severity score was 3.0 in flat midline versus 1.0 in flat right lateral $(\mathrm{P}=0.21)$. We obtained data for the following outcomes directly from trial authors: neonatal death -3 newborns (1 due to sepsis) in each group; any GM-IVH - 6 newborns in each group; and severe IVH - 1 newborn in each group.

\section{Excluded studies}

We excluded two trials (Antunes 2003; Wu 2015) because of characteristics of their interventions and populations. Antunes 2003 adopted the two different head positions at 48 hours of life, whereas in this review, we included trials in which the intervention was started within the first 48 hours of life (as specified in Types of interventions). We excluded Wu 2015 because mean birth weight was $>2.5 \mathrm{~kg}$, suggesting that included newborns were not of GA $\leq 32$ weeks, as specified in this review (Types of participants).

\section{Risk of bias in included studies}

We presented information on risk of bias in the included trials in the "Risk of bias graph" (Figure 2) and in the "Risk of bias summary" (Figure 3).

Figure 2. Risk of bias graph: review authors' judgements about each risk of bias item presented as percentages across all included studies.

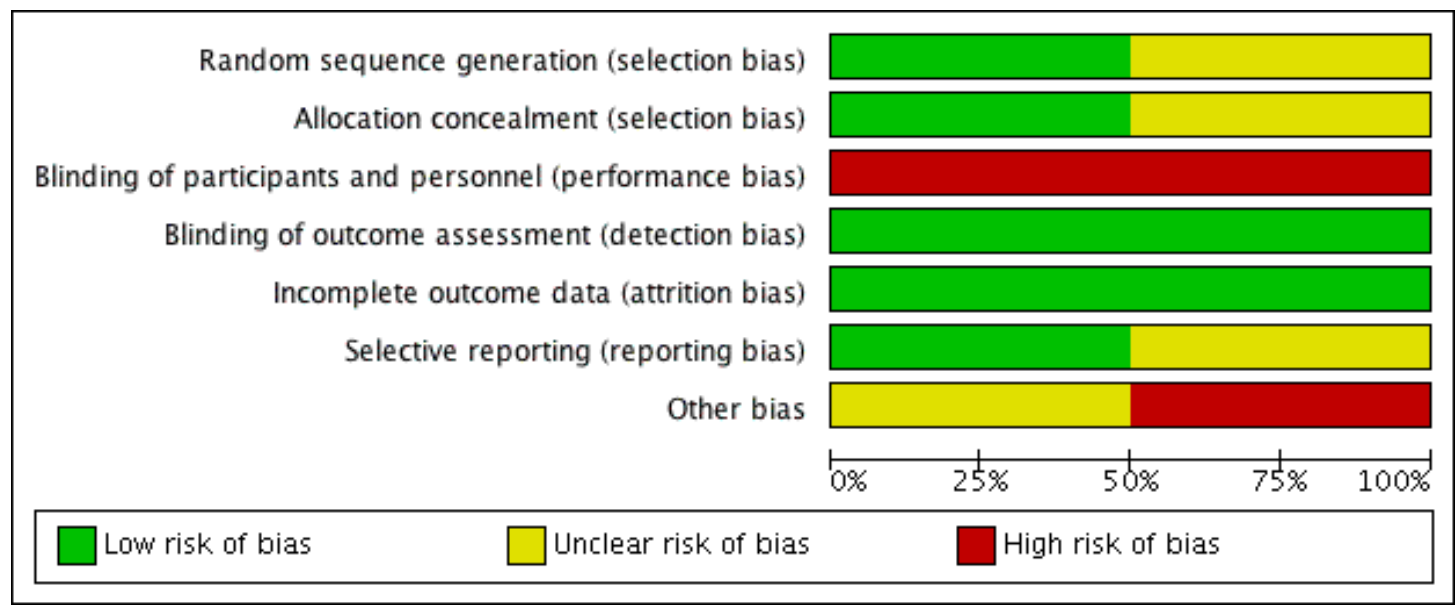


Figure 3. Risk of bias summary: review authors' judgements about each risk of bias item for each included study.

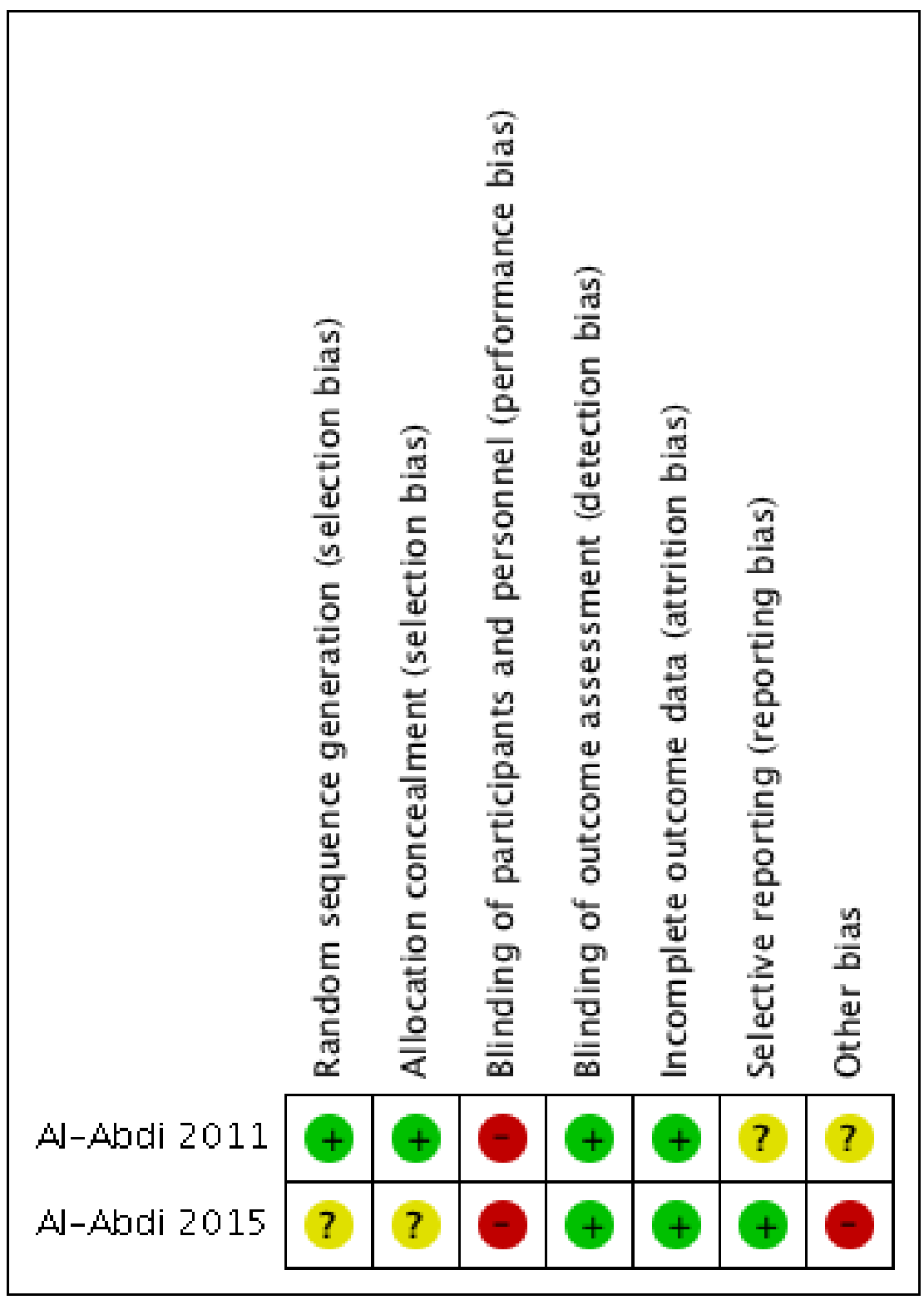

Head midline position for preventing the occurrence or extension of germinal matrix-intraventricular hemorrhage in preterm infants 


\section{Allocation}

Al-Abdi 2011 reported adequate sequence generation and randomization in blocks to ensure balanced combinations of positions and finally an identification number in a sealed envelope. Al-Abdi 2015 did not provide sufficient information on how the randomization sequence was generated and concealed, and we judged this study to be at unclear risk of selection bias.

\section{Blinding}

Owing to the nature of the intervention, blinding was not possible in the included studies; however, blinding was provided for outcome assessors.

\section{Incomplete outcome data}

The two studies reported outcomes for all randomized infants (no dropouts).

\section{Selective reporting}

The protocol was registered for Al-Abdi 2015 only. For Al-Abdi 2011 , we contacted study authors but found that the study protocol was not available.

\section{Other potential sources of bias}

Al-Abdi 2011 did not carry out calculation of sample size (pilot study). Al-Abdi 2015 performed the calculation but had premature stopping due to a low accrual rate (71 infants were enrolled instead of 600 , corresponding to $12 \%$ ).

\section{Effects of interventions}

See: Summary of findings for the main comparison Supine midline head position with the bed at $0^{\circ}$ versus supine head rotated $90^{\circ}$ with the bed at $0^{\circ}$ for preventing occurrence or extension of germinal matrix-intraventricular hemorrhage in preterm infants We planned to conduct multiple comparisons as described in Types of interventions. However, both included trials could be grouped in the first type of prespecified comparison - supine midline head position versus any other supine head position. More precisely, both trials compared supine midline head position with the bed at $0^{\circ}$ versus supine head rotated $90^{\circ}$ with the bed at $0^{\circ}$.

\section{Supine midline head position versus any other supine head position (comparison I)}

Two trials (Al-Abdi 2011; Al-Abdi 2015), with a total of 110 infants, met the eligibility criteria (see Summary of findings for the main comparison).

\section{Primary outcomes}

\section{GH-IVH: any severity (grade 1 to 4) (Outcome 1.1)}

See Analysis 1.1 and Figure 4.

Figure 4. Forest plot of comparison: I Supine midline head position with the bed at $0^{\circ}$ versus supine head rotated $90^{\circ}$ with the bed at $0^{\circ}$, outcome: I.I Intraventricular hemorrhage, any grade.

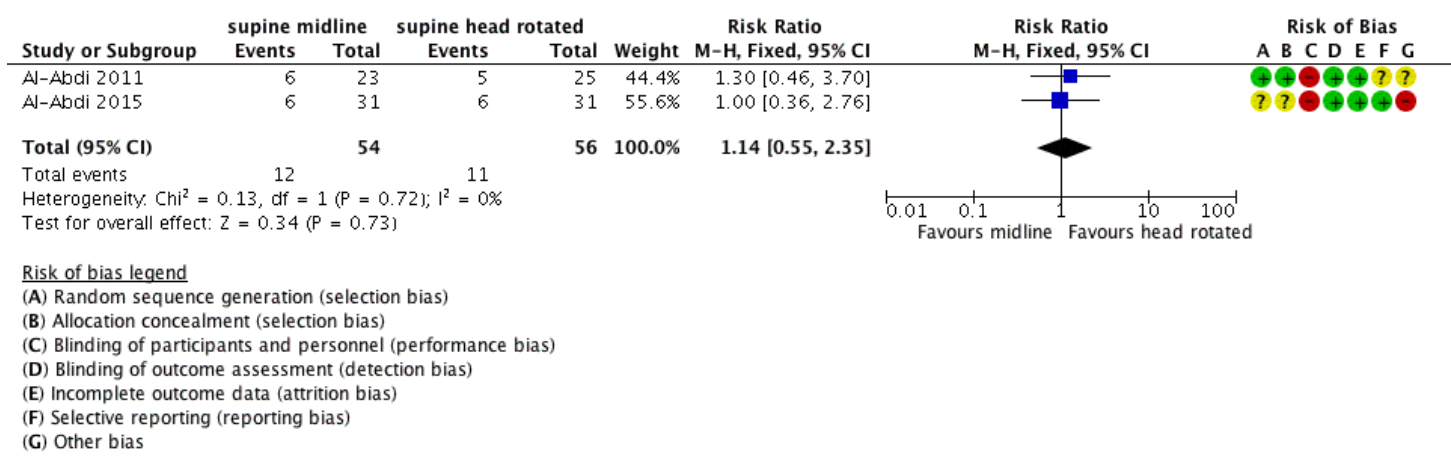

Head midline position for preventing the occurrence or extension of germinal matrix-intraventricular hemorrhage in preterm infants 
Both trials ( $\mathrm{n}=110$ infants) reported this outcome. Supine midline head position did not alter the risk of developing any GM-IVH (typical RR $1.14,95 \%$ CI 0.55 to 2.35 ; typical RD 0.03 , 95\% CI -0.13 to 0.18 ; two studies, 110 infants; $\mathrm{I}^{2}=0 \%$ for $\mathrm{RR}$ and $\mathrm{I}^{2}=$ $0 \%$ for $\mathrm{RD})$.

For Al-Abdi 2015, we obtained data for this outcome directly from trial authors.

Severe IVH: (grades 3 and 4) (Outcome 1.2)

See Analysis 1.2 and Figure 5.

Figure 5. Forest plot of comparison: I Supine midline head position with the bed at $0^{\circ}$ versus supine head rotated $90^{\circ}$ with the bed at $0^{\circ}$, outcome: 1.2 Intraventricular hemorrhage, grade 3 to 4.

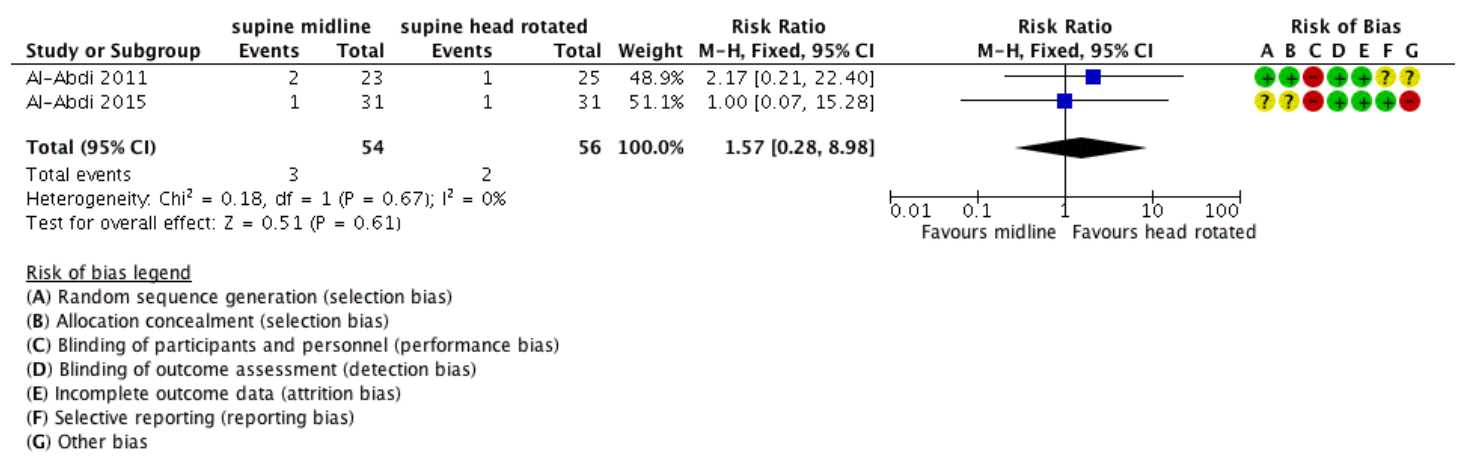

Both trials ( $\mathrm{n}=110$ infants) reported this outcome. Supine midline head position did not alter the risk of developing any severe IVH (typical RR $1.57,95 \%$ CI 0.28 to 8.98 ; typical RD 0.02 , $95 \%$ CI -0.06 to 0.10 ; two studies, 110 infants; $\mathrm{I}^{2}=0 \%$ for $\mathrm{RR}$ and $\mathrm{I}^{2}=$ $0 \%$ for RD).

For Al-Abdi 2015, we obtained data for this outcome directly from trial authors.

Neonatal mortality < 28 days of age (Outcome 1.3)

See Analysis 1.3 and Figure 6. 
Figure 6. Forest plot of comparison: I Supine midline head position with the bed at $0^{\circ}$ versus supine head rotated $90^{\circ}$ with the bed at $0^{\circ}$, outcome: 1.3 Neonatal mortality.

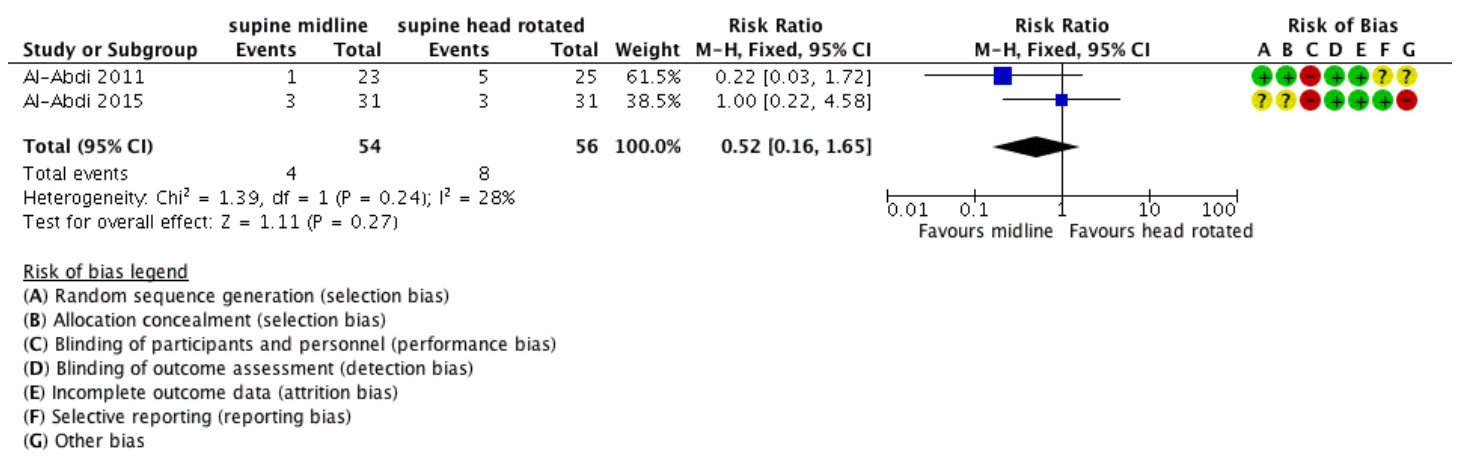

Both trials ( $\mathrm{n}=110$ infants) reported this outcome. Supine midline head position did not alter the risk of neonatal mortality (typical RR 0.52 , $95 \%$ CI 0.16 to 1.65 ; typical RD $-0.07,95 \%$ CI -0.18 to 0.05 ; two studies, 110 infants; $\mathrm{I}^{2}=28 \%$ for $\mathrm{RR}$ and $\mathrm{I}^{2}=44 \%$ for RD).

For both trials, we obtained data for this outcome directly from trial authors.

\section{Secondary outcomes}

Cystic periventricular leukomalacia (Outcome 1.4)

See Analysis 1.4.

One trial (Al-Abdi 2011) reported that 1 infant in the supine midline group $(1 / 23)$ versus no infants in the control group (0/ 25) received a diagnosis of cystic periventricular leukomalacia; this difference was not significant (RR 3.25, 95\% CI 0.14 to 76.01 ; $\mathrm{RD} 0.04,95 \% \mathrm{CI}-0.07$ to 0.15$)$. The test for heterogeneity was not applicable.

We obtained data for this outcome directly from trial authors.

\section{Retinopathy of prematurity, any stage (Outcome 1.5)}

See Analysis 1.5.

One trial (Al-Abdi 2011) reported a diagnosis of retinopathy of prematurity in 10 infants in the supine midline group (10/22) versus 4 infants in the control group (4/20); this difference was not significant (RR 2.27, 95\% CI 0.85 to 6.11 ; RD $0.25,95 \%$ CI -0.02 to 0.53$)$. The test for heterogeneity was not applicable. We obtained data for this outcome directly from trial authors.

Retinopathy of prematurity, $\geq$ stage 3 (Outcome 1.6)

See Analysis 1.6.
One trial (Al-Abdi 2011) reported that retinopathy of prematurity of stage $\geq 3$ was diagnosed in 3 infants in the supine midline group (3/22) versus 1 infant in the control group (1/20); this difference was not significant (RR 2.73, $95 \%$ CI 0.31 to 24.14 ; $\mathrm{RD} 0.09$, 95\% CI -0.09 to 0.26 ). The test for heterogeneity was not applicable.

We obtained data for this outcome directly from trial authors.

\section{Additional outcomes}

We found no data on the following: cerebellar hemorrhage; brain MRI abnormalities; impairment in cerebral hemodynamics; longterm neurodevelopmental outcomes; and major neurodevelopmental disability.

\section{Supine midline head position versus any other prone head position (comparison 2 )}

We found no trials comparing supine midline head position versus any other prone head position.

\section{Supine midline head position with the bed at $0^{\circ}$ versus supine midline head position with the bed tilted $\geq 30^{\circ}$ (comparison 3 )}

We found no trials comparing supine midline head position with the bed at $0^{\circ}$ versus supine midline head position with the bed tilted $\geq 30^{\circ}$.

\section{Subgroup analysis}

We were unable to conduct any of the planned subgroup analyses, as we included only two small trials. 


\section{I SCUSSION}

\section{Summary of main results}

We evaluated the efficacy of midline position for prevention of germinal matrix-intraventricular hemorrhage (GM-IVH) in very preterm infants. Only two trials (Al-Abdi 2011; Al-Abdi 2015), with a total of 110 preterm infants with gestational age less than 30 weeks, met the inclusion criteria of our review. Both trials compared supine midline head position versus supine head rotated $90^{\circ}$. One of the two trials was prematurely terminated owing to low accrual rate when only 71 neonates $(12 \%)$ had been recruited (Al-Abdi 2015). Results showed no statistically significant differences in the incidence or severity of GM-IVH and in mortality (primary outcomes of this review) between head midline and lateral position. However, given the imprecision of our estimates (i.e., wide confidence intervals [CIs]), results of this systematic review are consistent with a beneficial or detrimental effect of head midline positioning. When considering secondary outcomes, such as retinopathy of prematurity or cystic periventricular leukomalacia, we found no benefit of midline over lateral position. We identified one trial under Studies awaiting classification because most infants had a gestational age greater than that specified in the inclusion criteria of this review; moreover, study authors reported none of the outcomes specified in this review (Jalali 2012). We found no relevant studies on clinical trials registries for ongoing or recently completed trials.

\section{Overall completeness and applicability of evidence}

Available evidence is insufficient to show whether head midline position is an effective and safe intervention for preventing GM-IVH in very preterm neonates. Only two randomized trials (110 newborns) assessed this study question, and one of them was stopped early after recruiting only $12 \%$ of the planned sample size (Al-Abdi 2015). Both trials compared supine midline head position with the bed at $0^{\circ}$ versus supine head rotated $90^{\circ}$ with the bed at $0^{\circ}$ in infants with gestational age $(\mathrm{GA})<30$ weeks. We found no trials that compared supine versus prone midline head position, and no trials that compared effects of head tilting; thus we cannot draw any conclusions regarding their comparative effectiveness. Included trials enrolled few infants with GA $<26$ weeks - the group at highest risk for GM-IVH. Available data were insufficient for assessment of the primary outcome of this review as well as other important outcomes such as retinopathy of prematurity and longterm neurodevelopmental outcomes.

\section{Quality of the evidence}

According to the GRADE approach, we rated the overall quality of evidence for clinically relevant outcomes as "low" and "very low" (see Summary of findings for the main comparison). We downgraded the overall quality of evidence for critical outcomes because of (1) imprecision of results (small number of participants; few events) that could be a source of random error risk, and (2) premature stopping of participant enrollment. Random error is closely related to imprecision, as results of smaller studies are subject to greater sampling variation and hence are less precise (Higgins 2011). Given the nature of the intervention, it was not possible to blind personnel. The quality of the included trials, although they included small samples, was moderate in general and they showed low risk of bias (Figure 2 and Figure 3).

\section{Potential biases in the review process}

We used standard methods of the Cochrane Neonatal Review Group in conducting this systematic review. Our inclusive search strategy theoretically would have included all relevant studies. For example, availability of the full text was not imperative as in the systematic review on the same topic by de Bijl-Marcus and colleagues, which included one randomized controlled trial only (de Bijl-Marcus 2016). We minimized potential biases, although selection of criteria applied in considering studies for inclusion in this review (namely, Types of participants and Types of interventions) led to the exclusion of two trials (Antunes 2003; Wu 2015) - the former because the intervention was started after 48 hours of life, and the latter because included newborns were not of GA $\leq 32$ weeks, as specified in this review. Finally, we identified a trial under Studies awaiting classification because most infants had a gestational age greater than that specified in the inclusion criteria of this review (Jalali 2012).

\section{Agreements and disagreements with other studies or reviews}

Two other reviews have addressed the clinical question of the use of head midline position to prevent GM-IVH in very preterm neonates, with conflicting conclusions (Carteaux 2003; de Bijl-Marcus 2016). Carteaux and colleagues recommended implementing a plan of care that includes midline head positioning for premature infants (Carteaux 2003). The rationale for their decision - which was made before any randomized controlled trial had been conducted - is unclear, and we disagree with their conclusions. In contrast, another systematic review (de Bijl-Marcus 2016) was published after both trials by Al-Abdi (Al-Abdi 2011; Al-Abdi 2015) had been reported. However, Al-Abdi 2015 was published as an abstract only and therefore was not included in de Bijl-Marcus 2016, for which review authors specified that "availability of the full text was imperative." An additional, minor difference between our review and de Bijl-Marcus 2016 consists of assessment of reporting bias for Al-Abdi 2011, which we scored as unclear because the study protocol is not available. Moreover, 
we succeeded in contacting Dr. Al-Abdi to retrieve additional data on relevant outcomes for both trials (Al-Abdi 2011; Al-Abdi 2015). Overall, we agree with de Bijl-Marcus 2016 that evidence is insufficient to allow conclusions on effects of head positioning and/or tilting on the incidence of GMH-IVH in very preterm infants. It is noteworthy that regional cerebral saturations measured by near-infrared spectroscopy in extremely preterm infants during the first three days of life remained within normal range when the head was turned from the midline position to either side (Liao 2015). Other studies have compared the supine versus prone position in terms of decreased cerebral hemoglobin oxygenation and cerebral blood volume in supine versus prone position (Pichler 2001) and decreased volume of down-side ventricles versus volume of up-side ventricles (Nagdyman 1999). Nevertheless, these findings may not justify the recommendation - adopted in several neonatal intensive care units worldwide and endorsed by Carteaux 2003 - to maintain the head in midline position for prevention of GM-IVH. However, head and body position may have important effects on relevant outcomes such as pulmonary function. Two Cochrane systematic reviews have been published on the effects of body positioning on respiratory morbidity, that is, in spontaneously breathing (Bredemeyer 2012) and ventilated infants (Rivas-Fernandez 2016). In the review on body positioning for spontaneously breathing preterm infants with apnea, review authors reported that evidence is insufficient to show effects of body positioning on apnea, bradycardia, oxygen desaturation, and oxygen saturation (Bredemeyer 2012). However, the review on position of neonates receiving mechanical ventilation concluded that evidence of low to moderate quality favors the prone position for slightly improved oxygenation in neonates undergoing mechanical ventilation (Rivas-Fernandez 2016). However, these two reviews had a different objective, hence populations and interventions, as compared with those of this review, did not focus on GM-IVH.

\section{AUTHORS' CONCLUSIONS}

\section{Implications for practice}

We found very limited data on the effects of head midline position on germinal matrix-intraventricular hemorrhage and neonatal mortality in very preterm neonates. Both trials compared supine midline head position versus supine head rotated $90^{\circ}$ in infants with gestational age $<29$ weeks. Given the imprecision of our estimates, results of this systematic review are consistent with a beneficial or detrimental effect of head midline position, and do not provide a definitive answer to the review question. Limited evidence is available on other clinically relevant outcomes. The choice between prone and supine position might as well be based on available evidence from studies focusing on the impact of position on respiratory morbidity (Bredemeyer 2012; Rivas-Fernandez 2016).

\section{Implications for research}

Multiple studies have investigated different positions of the head and body of the preterm newborn, mainly focusing on effects on respiratory morbidity. Future trials should report on short- and long-term outcomes, including germinal matrix-intraventricular hemorrhage, cerebellar hemorrhage, cystic periventricular leukomalacia, retinopathy of prematurity, and neurodevelopmental disability. Populations might include term newborns as well, if a comprehensive approach to investigation of both neurological and respiratory outcomes is adopted. Supine midline head position might be compared with supine head rotated $90^{\circ}$, prone midline head position; or use of head tilting.

\section{ACKNOWLEDGEMENTS}

We thank Roger Soll for his precious advice, and Colleen Ovelman and Yolanda Brosseau for their kind and efficient support.

\section{REFERENCES}

\section{References to studies included in this review}

\section{Al-Abdi 2011 \{published data only\}}

Al-Abdi SY, Nojoom MS, Alshaalan HM, Al-Aamri MA. Pilot-randomized study on intraventricular hemorrhage with midline versus lateral head positions. Saudi Medical Journal 2011;32(4):420-1.

Al-Abdi 2015 \{published data only\} Al-Abdi S, Alallah J, Al Omran A, Al Alwan Q, Al Hashimi $\mathrm{H}$, Haidar S. The risk of intraventricular hemorrhage with flat midline versus flat right lateral head positions: a prematurely terminated multicenter randomized clinical trial. The Pediatric Academic Societies (PAS). 2015. https:/

/clinicaltrials.gov/ct2/show/NCT01584375]

\section{References to studies excluded from this review}

Antunes 2003 \{published data only\}

Antunes LC, Rugolo LM, Crocci AJ. Effect of preterm infant position on weaning from mechanical ventilation [Efeito da posição do prematuro no desmame da ventilação mecânica]. Jornal de Pediatria 2003;79(3):239-44.

Wu 2015 \{published data only\}

Wu J, Zhai J, Jiang H, Sun Y, Jin B, Zhang Y, et al. Effect of change of mechanical ventilation position on the 
treatment of neonatal respiratory failure. Cell Biochemistry and Biophysics 2015;72(3):845-9.

\section{References to studies awaiting assessment}

Jalali 2012 \{published data only\}

Jalali SZ, Mojtabaei SH, Heidarzadeh A, Aghamahdi F, Ahmad-Soltani M. The influence of lateral and supine position on bacterial colonization of endotracheal tube in neonates admitted to neonatal intensive care unit. Iranian Journal of Pediatrics 2012;22(4):499-504.

\section{Additional references}

\section{Ancora 2010}

Ancora G, Maranella E, Aceti A, Pierantoni L, Grandi $S$, Corvaglia L, et al. Effect of posture on brain hemodynamics in preterm newborns not mechanically ventilated. Neonatology 2010;97(3):212-7. [DOI: 10.1159/000253149; PUBMED: 19887848

\section{Ballabh 2014}

Ballabh P. Pathogenesis and prevention of intraventricular hemorrhage. Clinics in Perinatology 2014;41(1):47-67. [DOI: 10.1016/j.clp.2013.09.007; PUBMED: 24524446

\section{Barradas 2006}

Barradas J, Fonseca A, Guimarães CL, Lima GM. Relationship between positioning of premature infants in Kangaroo Mother Care and early neuromotor development. Jornal de Pediatria 2006;82(6):475-80. [DOI: 10.2223/ JPED.1565; PUBMED: 17171208

Bayley 1993

Bayley N. Bayley Scales of Infant Development. 2nd Edition. San Antonio, TX: The Psychological Corporation, 1993.

Bayley 2006

Bayley N. Bayley Scales of Infant and Toddler Development. 3rd Edition. San Antonio, TX: Harcourt Assessment, 2006.

Bembich 2012

Bembich S, Oretti C, Travan L, Clarici A, Massaccesi $S$, Demarini $S$. Effects of prone and supine position on cerebral blood flow in preterm infants. Journal of Pediatrics 2012;160(1):162-4. [DOI: 10.1016/j.jpeds.2011.08.056; PUBMED: 22000305

Bredemeyer 2012

Bredemeyer SL, Foster JP. Body positioning for spontaneously breathing preterm infants with apnoea. Cochrane Database of Systematic Reviews 2012, Issue 6. [DOI: 10.1002/14651858.CD004951.pub2

\section{Canadian Neonatal Network 2014}

The Canadian Neonatal Network ${ }^{\mathrm{TM}}$. Annual Report 2014. Written and prepared by Dr Prakesh Shah, Eugene W. Yoon, Priscilla Chan and Members of the Annual Report Review Committee. http://www.canadianneonatalnetwork.org.

Carteaux 2003

Carteaux P, Cohen H, Check J, George J, McKinley P, Lewis W, et al. Evaluation and development of potentially better practices for the prevention of brain hemorrhage and ischemic brain injury in very low birth weight infants. Pediatrics 2003;11(4 Pt 2):e489-96. [PUBMED: 12671169]

\section{Cochrane EPOC Group 2015}

Effective Practice, Organisation of Care (EPOC). Data extraction and management. EPOC resources for review authors. Oslo: Norwegian Knowledge Centre for the Health Services, 2015. http://epoc.cochrane.org/epocspecific-resources-review-authors.

\section{Cornette 2002}

Cornette LG, Tanner SF, Ramenghi LA, Miall LS, Childs AM, Arthur RJ, et al. Magnetic resonance imaging of the infant brain: anatomical characteristics and clinical significance of punctate lesions. Archives of Disease in Childhood. Fetal and Neonatal Edition 2002;86(3):F171-7. [PUBMED: 11978747]

\section{Cowan 1985}

Cowan F, Thoresen M. Changes in superior sagittal sinus blood velocities due to postural alterations and pressure on the head of the newborn infant. Pediatrics 1985;75(6): 1038-47. [PUBMED: 3889817]

\section{Cowan 1987}

Cowan F, Thoresen M. The effect of intermittent positive pressure ventilation on cerebral arterial and venous blood velocities in the newborn infant. Acta Paediatrica Scandinavica 1987;76(2):239-47. [PUBMED: 3296626]

Dani 2007

Dani C, Bertini G, Cecchi A, Corsini I, Pratesi S, Rubaltelli FF. Brain hemodynamic effects of nasal continuous airway pressure in preterm infants of less than 30 weeks' gestation. Acta Pediatrica 2007;96(10):1421-5. [DOI: 10.1111/ j.1651-2227.2007.00453.x; PUBMED: 17714539

\section{de Bijl-Marcus 2016}

de Bijl-Marcus KA, Brouwer AJ, de Vries LS, van WezelMeijler G. The effect of head positioning and head tilting on the incidence of intraventricular hemorrhage in very preterm infants: a systematic review. Neonatology 2016; 111(3):267-79. [DOI: 10.1159/000449240; PUBMED: 27923236

\section{Dolfin 1983}

Dolfin T, Skidmore MB, Fong KW, Hoskins EM, Shennan AT. Incidence, severity, and timing of subependymal and intraventricular hemorrhages in preterm infants born in a perinatal unit as detected by serial real-time ultrasound. Pediatrics 1983;71(4):541-6. [PUBMED: 6835737]

\section{Egger 1997}

Egger M, Davey Smith G, Schneider M, Minder C. Bias in meta-analysis detected by a simple, graphical test. BMJ 1997;315(7109):629-34. [PUBMED: 9310563]

\section{Emery 1983}

Emery JR, Peabody JL. Head position affects intracranial pressure in newborn infants. Journal of Pediatrics 1983;103 (6):950-3. [PUBMED: 6644434]

\section{Fumagalli 2009} Fumagalli M, Ramenghi LA, Righini A, Groppo M, Bassi L, De Carli A, et al. Cerebellar hemorrhages and pons development in extremely low birth weight infants. Frontiers 
in Bioscience (Elite Edition) 2009;1:537-41. [PUBMED: 19482668]

GRADEpro GDT [Computer program]

GRADE Working Group, McMaster University. GRADEpro [www.gradepro.org]. Version 14 September 2016. Hamilton (ON): GRADE Working Group, McMaster University, 2014.

\section{Graça 2013}

Graça AM, Geraldo AF, Cardoso K, Cowan FM. Preterm cerebellum at term age: ultrasound measurements are not different from infants born at term. Pediatric Research 2013; 74(6):698-704. [DOI: 10.1038/pr.2013.154; PUBMED: 24002327

Griffiths 1954

Griffiths R. The Abilities of Babies: A Study in Mental Measurement. New York, NY: McGraw-Hill, 1954.

\section{Hamrick 2004}

Hamrick SE, Miller SP, Leonard C, Glidden DV, Goldstein $\mathrm{R}$, Ramaswamy V, et al. Trends in severe brain injury and neurodevelopmental outcome in premature newborn infants: the role of cystic periventricular leukomalacia. Journal of Pediatrics 2004;145(5):593-9. [DOI: 10.1016/ j.jpeds.2004.05.042; PUBMED: 15520756

\section{Higgins 2003}

Higgins JP, Thompson SG, Deeks JJ, Altman DG. Measuring inconsistency in meta-analyses. BMJ 2003; 327(7414):557-60. [DOI: 10.1136/bmj.327.7414.557; PUBMED: 12958120

\section{Higgins 2011}

Higgins JPT, Green S (editors). Cochrane Handbook for Systematic Reviews of Interventions, Version 5.1.0 [updated March 2011]. The Cochrane Collaboration, 2011. handbook.cochrane.org.

\section{Horbar 2002}

Horbar JD, Badger GJ, Carpenter JH, Fanaroff AA, Kilpatrick S, LaCorte M, et al. Members of the Vermont Oxford Network. Trends in mortality and morbidity for very low birth weight infants, 1991-1999. Pediatrics 2002; 110(1 Pt 1):143-51. [PUBMED: 12093960]

\section{ICROP 1984}

International Committee for the Classification of Retinopathy of Prematurity. An international classification of retinopathy of prematurity. Pediatrics 1984;74(1): 127-33. [PUBMED: 6547526]

\section{Jacobs 2013}

Jacobs SE, Berg M, Hunt R, Tarnow-Mordi WO, Inder TE, Davis PG. Cooling for newborns with hypoxic ischaemic encephalopathy. Cochrane Database of Systematic Reviews 2013, Issue 1. [DOI: 10.1002/14651858.CD003311.pub3; PUBMED: 23440789

Leahy 1982

Leahy FA, Durand M, Cates D, Chernick V. Cranial blood volume changes during mechanical ventilation and spontaneous breathing in newborn infants. Journal of Pediatrics 1982;101(6):984-7. [PUBMED: 7143179]
Liao 2015

Liao SM, Rao R, Mathur AM. Head position change is not associated with acute changes in bilateral cerebral oxygenation in stable preterm infants during the first 3 days of life. American Journal of Perinatology 2015;32(7):645-52. [DOI: 10.1055/s-0034-1390348; PUBMED: 25282608

Limperopoulos 2007

Limperopoulos C, Bassan H, Gauvreau K, Robertson RL Jr, Sullivan NR, Benson CB, et al. Does cerebellar injury in premature infants contribute to the high prevalence of long-term cognitive, learning, and behavioral disability in survivors?. Pediatrics 2007;120(3):584-93. [DOI: 10.1542/peds.2007-1041; PUBMED: 17766532

\section{Malusky 2011}

Malusky S, Donze A. Neutral head positioning in premature infants for intraventricular hemorrhage prevention: an evidence-based review. Neonatal Network NN 2011;30(6): 381-96. [DOI: 10.1891/0730-0832.30.6.381; PUBMED: 22052118

\section{McLendon 2003}

McLendon D, Check J, Carteaux P, Michael L, Moehring J, Secrest JW, et al. Implementation of potentially better practices for the prevention of brain hemorrhage and ischemic brain injury in very low birth weight infants. Pediatrics 2003;111(4 Pt 2):e497-503. [PUBMED: 12671170]

\section{Moritz 2008}

Moritz B, Fritz M, Mann C, Simma B. Nasal continuous positive airway pressure (nCPAP) does not change cardiac output in preterm infants. American Journal of Perinatology 2008;25(2):105-9. [DOI: 10.1055/s-2008-1040341; PUBMED: 18240105

\section{Nagdyman 1999}

Nagdyman N, Walka MM, Kampmann W, Stöver B, Obladen M. 3-D ultrasound quantification of neonatal cerebral ventricles in different head positions. Ultrasound in Medicine and Biology 1999;25(6):895-900. [PUBMED: 10461716]

Nakamura 1990

Nakamura Y, Okudera T, Fukuda S, Hashimoto T. Germinal matrix hemorrhage of venous origin in preterm neonates. Human Pathology 1990;21(10):1059-62. [PUBMED: 2210729]

\section{Nankervis 2010}

Nankervis CA, Martin EM, Crane ML, Samson KS, Welty SE, Nelin LD. Implementation of a multidisciplinary guideline-driven approach to the care of the extremely premature infant improved hospital outcomes. Acta Paediatrica 2010;99(2):188-93. [DOI: 10.1111/ j.1651-2227.2009.01563.x; PUBMED: 19863632

\section{Noori 2014}

Noori S, McCoy M, Anderson MP, Ramji F, Seri I. Changes in cardiac function and cerebral blood flow in relation to peri/intraventricular hemorrhage in extremely preterm infants. Journal of Pediatrics 2014;164(2):264-70.e1- 
3. [DOI: 10.1016/j.jpeds.2013.09.045; PUBMED: 24183212

\section{Obladen 2008}

Obladen M, Metze B, Henrich W, Aktas A, Czernik C, Schulz-Baldes A. Interdisciplinary surveillance of intraventricular hemorrhage associated conditions in infants <1000 g. Acta Paediatrica 2008;97(6):731-7. [DOI: 10.1111/j.1651-2227.2008.00812.x; PUBMED: 18460106

Osborn 2003

Osborn DA, Evans N, Kluckow M. Hemodynamic and antecedent risk factors of early and late periventricular/ intraventricular hemorrhage in premature infants. Pediatrics 2003;112(1 Pt 1):33-9. [PUBMED: 12837865]

Pape 1979

Pape KE, Wigglesworth JS. Haemorrhage, Ischaemia and the Perinatal Brain. Cambridge, UK: Cambridge University Press, 1979.

Papile 1978

Papile LA, Burstein J, Burstein R, Koffler H. Incidence and evolution of subependymal and intraventricular hemorrhage: a study of infants with birth weights less than 1,500 gm. Journal of Pediatrics 1978;92(4):529-34. [PUBMED: 305471]

Parodi 2015

Parodi A, Morana G, Severino MS, Malova M, Natalizia AR, Sannia A, et al. Low-grade intraventricular hemorrhage: is ultrasound good enough?. Journal of Maternal-Fetal \& Neonatal Medicine 2015;28(Suppl 1):2261-4. [DOI: 10.3109/14767058.2013.796162; PUBMED: 23968243

Pellicer 2002

Pellicer A, Gaya F, Madero R, Quero J, Cabanas F. Noninvasive continuous monitoring of the effects of the head position on brain hemodynamic in ventilated infants. Pediatrics 2002;109(3):434-40. [PUBMED: 11875138]

Philip 1989

Philip AG, Allan WC, Tito AM, Wheeler LR. Intraventricular hemorrhage in preterm infants: declining incidence in the 1980s. Pediatrics 1989;84(5):797-801. [PUBMED: 2677959]

Pichler 2001

Pichler G, Schmölzer G, Müller W, Urlesberger B. Body position-dependent changes in cerebral hemodynamics during apnea in preterm infants. Brain \& Development 2001;23(6):395-400. [PUBMED: 11578850]

RevMan 2014 [Computer program]

Nordic Cochrane Centre, The Cochrane Collaboration. Review Manager 5 (RevMan 5). Version 5.3. Copenhagen:
Nordic Cochrane Centre, The Cochrane Collaboration, 2014.

\section{Rivas-Fernandez 2016}

Rivas-Fernandez M, Roqué i Figuls M, Diez-Izquierdo A, Escribano J, Balaguer A. Infant position in neonates receiving mechanical ventilation. Cochrane Database of Systematic Reviews 2016, Issue 11. [DOI: 10.1002/ 14651858.CD003668.pub4; PUBMED: 27819747

\section{Rutherford 2010}

Rutherford MA, Supramaniam V, Ederies A, Chew A, Bassi L, Groppo M, et al. Magnetic resonance imaging of white matter diseases of prematurity. Neuroradiology 2010;52(6): 505-21. [DOI: 10.1007/s00234-010-0700-y; PUBMED: 20422407

\section{Schünemann 2013}

Schünemann H, Broż ek J, Guyatt G, Oxman A, editors. GRADE Working Group. GRADE Handbook for Grading Quality of Evidence and Strength of Recommendations. www.guidelinedevelopment.org/handbook. Updated October 2013.

\section{Sherlock 2005}

Sherlock RL, Anderson PJ, Doyle LW, Victorian Infant Collaborative Study Group. Neurodevelopmental sequelae of intraventricular hemorrhage at 8 years of age in a regional cohort of ELBW/very preterm infants. Early Human Development 2005;81(11):909-16. [DOI: 10.1016/ j.earlhumdev.2005.07.007; PUBMED: 16126353

\section{Takashima 1978}

Takashima S, Tanaka K. Microangiography and vascular permeability of the subependymal matrix in the premature infant. Canadian Journal of Neurological Sciences [Journal Canadien des Sciences Neurologiques] 1978;5(1):45-50. [PUBMED: 647497]

\section{Takashima 2009}

Takashima S, Itoh M, Oka A. A history of our understanding of cerebral vascular development and pathogenesis of perinatal brain damage over the past 30 years. Seminars in Pediatric Neurology 2009;16(4):226-36. [DOI: 10.1016/ j.spen.2009.09.004; PUBMED: 19945657

Vermont Oxford Network 2013 Vermont Oxford Network. Database of very low birth weight infants born in 2012. Nightingale Internet Reporting System. http://public.vtoxford.org (accessed 4 April 2014)

Volpe 2008

Volpe JV. Neurology of the Newborn. 5th Edition. Philadelphia, PA: Saunders, 2008.

* Indicates the major publication for the study 


\section{CHARACTERISTICS OF STUDIES}

\section{Characteristics of included studies [ordered by study ID]}

Al-Abdi 2011

Methods

Randomized parallel clinical trial, conducted at King Abdulaziz Hospital, Al-Ahsa, Saudi Arabia, and including 48 preterm infants in 2008-2009. Randomization was stratified on the basis of gender and gestational age ( $<27$ or 27 to $29+6$ weeks). Randomization was carried out according to a predetermined computer-generated randomization sequence with consecutively numbered sealed envelopes. In the case of eligible multiple births, each infant was randomly assigned. An intention-to-treat analysis was used throughout this study. Among infants in the head midline group, 12 were cared for in a left-tilted and 13 in a right-tilted midline head position

Participants

Preterm infants were enrolled in the trial if they met the following criteria: (1) inborn; (2) gestational age $<30$ weeks; and (3) postnatal age $<2$ hours. Exclusion criteria included the presence of lethal congenital anomalies, hypoxic ischemic encephalopathy, and the need for full cardiopulmonary resuscitation at birth

Interventions Infants lying on beds at $0^{\circ}$ were randomly assigned to be cared for in a supine midline (23 infants) or supine lateral ( 25 infants) head position. In the supine midline position, the infant's chin was kept at a $90^{\circ}$ angle to the bed. In the lateral head position, the head was tilted $90^{\circ}$ to either side. At enrollment, it was left to the bedside nurse to place the head in the right-tilted or left-titled position

Outcomes

Primary outcome: rate of intraventricular hemorrhage of all grades

Secondary outcomes: rate of intraventricular hemorrhage, grade 3 to 4; neonatal mortality; retinopathy of prematurity (any stage; $\geq$ stage 3); cystic periventricular leukomalacia

Notes

This was a pilot study (underpowered)

\section{Risk of bias}

\begin{tabular}{l|l|l}
\hline Bias & Authors' judgement & Support for judgement \\
\hline $\begin{array}{l}\text { Random sequence generation (selection } \\
\text { bias) }\end{array}$ & Low risk & $\begin{array}{l}\text { Randomization was carried out accord- } \\
\text { ing to a predetermined computer-gener- } \\
\text { ated randomization sequence }\end{array}$ \\
\hline $\begin{array}{l}\text { Allocation concealment (selection bias) } \\
\text { Llinding of participants and personnel } \\
\text { (performance bias) }\end{array}$ & Low risk & $\begin{array}{l}\text { Envelopes for randomization were opaque } \\
\text { (we obtained data for this outcome directly } \\
\text { from trial authors) }\end{array}$ \\
\hline & & Unblinded intervention \\
\hline
\end{tabular}

Head midline position for preventing the occurrence or extension of germinal matrix-intraventricular hemorrhage in preterm infants 
Al-Abdi 2011 (Continued)

\begin{tabular}{l|l|l}
\hline $\begin{array}{l}\text { Blinding of outcome assessment (detection } \\
\text { bias) } \\
\text { All outcomes }\end{array}$ & Low risk & $\begin{array}{l}\text { Head ultrasound scans were assessed by } 2 \\
\text { radiologists who were blinded to assign- } \\
\text { ment of head position }\end{array}$ \\
\hline $\begin{array}{l}\text { Incomplete outcome data (attrition bias) } \\
\text { All outcomes }\end{array}$ & Low risk & No missing outcome data \\
\hline Selective reporting (reporting bias) & Unclear risk & Study protocol not available \\
\hline Other bias & Unclear risk & Trial was underpowered (pilot) \\
\hline
\end{tabular}

\section{Al-Abdi 2015}

\begin{tabular}{ll} 
Methods & $\begin{array}{l}\text { Multicenter randomized (block size of } 4) \text { parallel clinical trial. Head ultrasound scan was } \\
\text { performed within } 12 \text { hours of life, at } 168 \text { hours of life, and at the physician's discretion. } \\
\text { Head ultrasound scan was reported by } 3 \text { radiologists who were blinded to assignment of } \\
\text { head position. The primary analysis was by intention to treat }\end{array}$ \\
\hline Participants & $\begin{array}{l}\text { Preterm neonates }(<30 \text { weeks' gestation) without intraventricular hemorrhage at } 12 \\
\text { hours of life scan } \\
\text { Exclusion criteria: outborn; lethal congenital anomalies; hypoxic ischemic encephalopa- } \\
\text { thy; external cardiac compression or epinephrine administration at birth }\end{array}$ \\
\hline Interventions & $\begin{array}{l}\text { Heads of } 62 \text { preterm neonates were randomly placed in flat midline (n }=31) \text { or flat right } \\
\text { lateral position }(\mathrm{n}=31) \text { throughout the first } 168 \text { hours of life }\end{array}$ \\
\hline Outcomes & $\begin{array}{l}\text { The only outcome reported in the abstract was intraventricular hemorrhage } \\
\text { Notes }\end{array}$ \\
\hline $\begin{array}{l}\text { Although the planned sample size was } 600 \text { (alpha } 5 \% \text {; power } 80 \%), \text { the study was } \\
\text { prematurely terminated owing to low accrual rate when only } 71 \text { neonates }(12 \%) \text { had } \\
\text { been recruited } \\
\text { Trial was registered on https://clinicaltrials.gov/ct } 2 / \text { show/NCT01584375 and findings } \\
\text { presented at the Pediatric Academic Societies (PAS) in 2015 }\end{array}$ \\
\hline
\end{tabular}

\section{Risk of bias}

\begin{tabular}{l|l|l}
\hline Bias & Authors' judgement & Support for judgement \\
\hline $\begin{array}{l}\text { Random sequence generation (selection } \\
\text { bias) }\end{array}$ & Unclear risk & Method of randomization not stated \\
\hline $\begin{array}{l}\text { Allocation concealment (selection bias) } \\
\text { Blinding of participants and personnel } \\
\text { (performance bias) } \\
\text { All outcomes }\end{array}$ & High risk & Method of allocation not stated \\
\hline
\end{tabular}

Head midline position for preventing the occurrence or extension of germinal matrix-intraventricular hemorrhage in preterm infants 


\section{Al-Abdi 2015 (Continued)}

\begin{tabular}{|c|c|c|}
\hline $\begin{array}{l}\text { Blinding of outcome assessment (detection } \\
\text { bias) } \\
\text { All outcomes }\end{array}$ & Low risk & $\begin{array}{l}\text { Head ultrasound scans were reported by } \\
3 \text { radiologists who were blinded to assign- } \\
\text { ment of head position }\end{array}$ \\
\hline $\begin{array}{l}\text { Incomplete outcome data (attrition bias) } \\
\text { All outcomes }\end{array}$ & Low risk & No missing outcome data \\
\hline Selective reporting (reporting bias) & Low risk & Study protocol available \\
\hline Other bias & High risk & $\begin{array}{l}\text { Trial lacks power because only } 71 \text { infants } \\
\text { were enrolled (instead of } 600 \text { ) }\end{array}$ \\
\hline
\end{tabular}

\section{Characteristics of excluded studies [ordered by study ID]}

\begin{tabular}{l|l}
\hline Study & Reason for exclusion \\
\hline Antunes 2003 & Intervention started after 48 hours of life \\
\hline Wu 2015 & $\begin{array}{l}\text { Mean birth weight was }>2.5 \mathrm{~kg}(2.75 \pm 0.58 \text { in intervention group and } 2.92 \pm 0.64 \text { in control group }) \text {, suggesting } \\
\text { that included newborns were not } \leq 32 \text { weeks' gestational age as specified in this review }\end{array}$ \\
\hline
\end{tabular}

\section{Characteristics of studies awaiting assessment [ordered by study ID]}

\section{Jalali 2012}

Methods Randomized clinical trial conducted at 17 Shahrivar Children's Hospital of Rasht (Rasht Iran), including 31 intubated newborns in the period 2010-2011

Participants Inclusion criteria: gestational age $\geq 28$ weeks, tracheal intubation at postnatal age $<48$ hours, absence of congenital malformations

Patients were excluded if they were given a diagnosis of congenital sepsis or pneumonia, or could not be maintained in mechanical ventilation for 5 days

Interventions Neonates were randomized to be nursed in supine $(\mathrm{n}=16)$ or lateral position $(\mathrm{n}=15)$

Outcomes No outcomes specified in our review were reported by study authors

Notes The objective of this trial was to evaluate the influence of lateral and supine position on bacterial colonization of the endotracheal tube in ventilated neonates. Approximately half of the included infants had a gestational age greater than that specified in the inclusion criterion of this review. We tried to contact trial authors but received no response. Study authors reported on none of the outcomes specified in this review 


\section{DATA A D A NALYES}

Comparison 1. Supine midline head position with the bed at $0^{\circ}$ versus supine head rotated $90^{\circ}$ with the bed at $0^{\circ}$

\begin{tabular}{|c|c|c|c|c|}
\hline Outcome or subgroup title & $\begin{array}{l}\text { No. of } \\
\text { studies }\end{array}$ & $\begin{array}{c}\text { No. of } \\
\text { participants }\end{array}$ & Statistical method & Effect size \\
\hline $\begin{array}{l}1 \text { Intraventricular hemorrhage, } \\
\text { any grade }\end{array}$ & 2 & 110 & Risk Ratio (M-H, Fixed, 95\% CI) & $1.14[0.55,2.35]$ \\
\hline $\begin{array}{l}2 \text { Intraventricular hemorrhage, } \\
\text { grade } 3 \text { to } 4\end{array}$ & 2 & 110 & Risk Ratio (M-H, Fixed, 95\% CI) & $1.57[0.28,8.98]$ \\
\hline 3 Neonatal mortality & 2 & 110 & Risk Ratio (M-H, Fixed, 95\% CI) & $0.52[0.16,1.65]$ \\
\hline $\begin{array}{l}4 \text { Cystic periventricular } \\
\text { leukomalacia }\end{array}$ & 1 & 48 & Risk Ratio (M-H, Fixed, 95\% CI) & $3.25[0.14,76.01]$ \\
\hline $\begin{array}{l}5 \text { Retinopathy of prematurity, any } \\
\text { stage }\end{array}$ & 1 & 42 & Risk Ratio (M-H, Fixed, 95\% CI) & $2.27[0.85,6.11]$ \\
\hline $\begin{array}{l}6 \text { Retinopathy of prematurity, } \geq \\
\text { stage } 3\end{array}$ & 1 & 42 & Risk Ratio (M-H, Fixed, 95\% CI) & $2.73[0.31,24.14]$ \\
\hline
\end{tabular}

\section{Analysis I.I. Comparison I Supine midline head position with the bed at $0^{\circ}$ versus supine head rotated $90^{\circ}$ with the bed at $0^{\circ}$, Outcome I Intraventricular hemorrhage, any grade.}

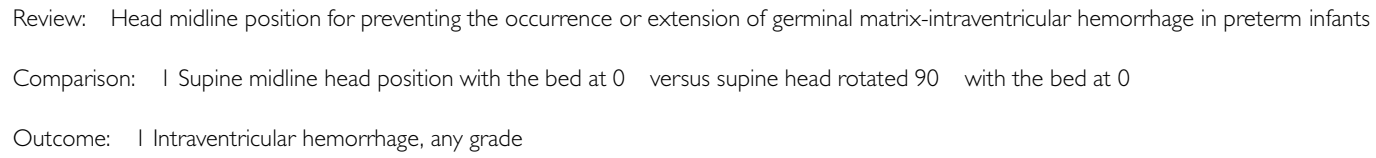

$6 / 31+55 \%$


Analysis I.2. Comparison I Supine midline head position with the bed at $0^{\circ}$ versus supine head rotated $90^{\circ}$ with the bed at $0^{\circ}$, Outcome 2 Intraventricular hemorrhage, grade 3 to 4 .

Review: Head midline position for preventing the occurrence or extension of germinal matrix-intraventricular hemorrhage in preterm infants

Comparison: I Supine midline head position with the bed at 0 versus supine head rotated 90 with the bed at 0

Outcome: 2 Intraventricular hemorrhage, grade 3 to 4

\begin{tabular}{|c|c|c|c|c|c|}
\hline \multirow[t]{2}{*}{ Study or subgroup } & supine midline & supine head rotated & Risk Ratio & Weight & Risk Ratio \\
\hline & $n / N$ & $\mathrm{n} / \mathrm{N}$ & M-H,Fixed,95\% Cl & & M-H,Fixed,95\% Cl \\
\hline Al-Abdi 20l I & $2 / 23$ & $1 / 25$ & & $48.9 \%$ & $2.17[0.21,22.40]$ \\
\hline Al-Abdi 2015 & $|/ 3|$ & $|/ 3|$ & & $51.1 \%$ & $1.00[0.07,15.28]$ \\
\hline
\end{tabular}

Total (95\% CI)

54

56

$100.0 \%$

$1.57[0.28,8.98$ ]

Total events: 3 (supine midline), 2 (supine head rotated)

Heterogeneity: $\mathrm{Chi}^{2}=0.18, \mathrm{df}=\mathrm{I}(\mathrm{P}=0.67) ; \mathrm{I}^{2}=0.0 \%$

Test for overall effect: $Z=0.5 \mathrm{I}(P=0.6 \mathrm{I})$

Test for subgroup differences: Not applicable

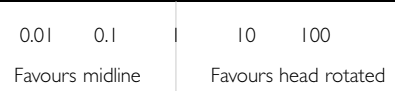

Analysis I.3. Comparison I Supine midline head position with the bed at $0^{\circ}$ versus supine head rotated $90^{\circ}$ with the bed at $0^{\circ}$, Outcome 3 Neonatal mortality.

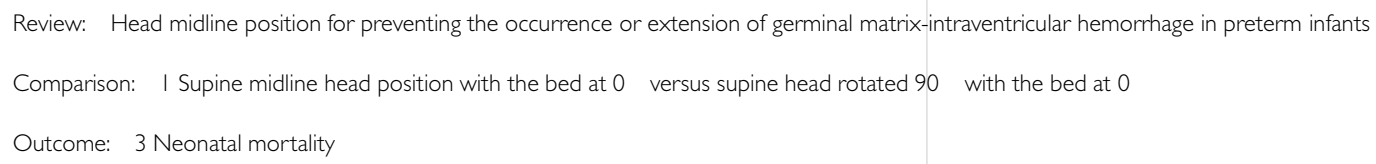

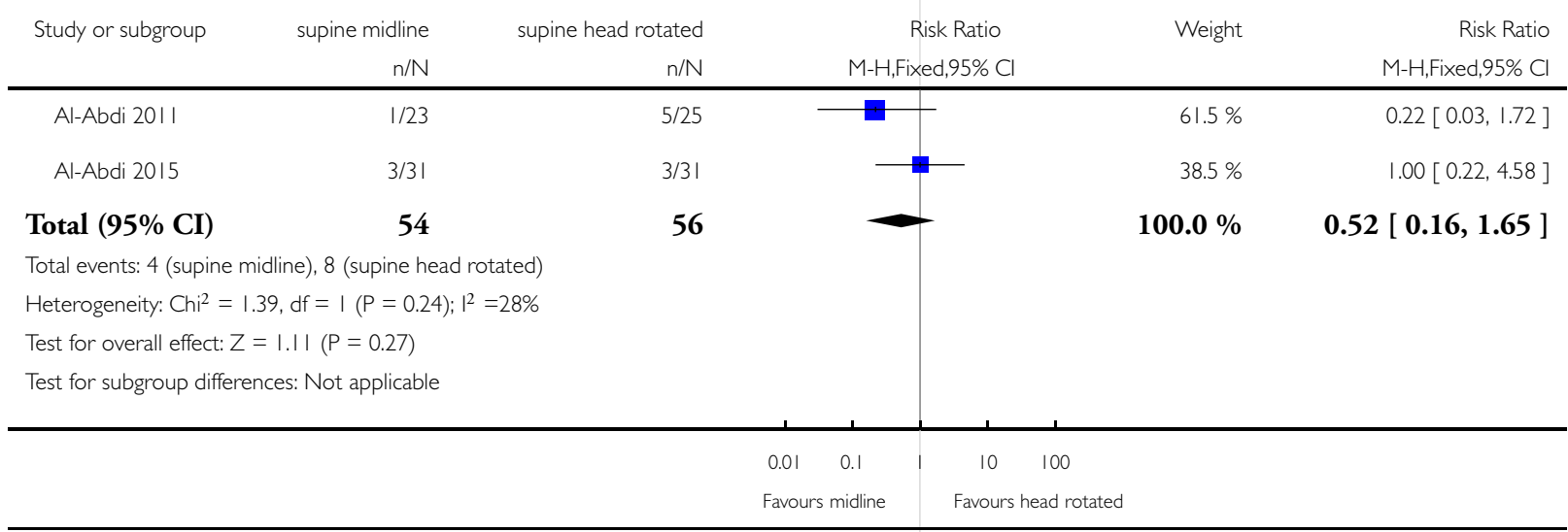

Head midline position for preventing the occurrence or extension of germinal matrix-intraventricular hemorrhage in preterm infants 30 (Review)

Copyright $(2017$ The Cochrane Collaboration. Published by John Wiley \& Sons, Ltd. 
Analysis I.4. Comparison I Supine midline head position with the bed at $0^{\circ}$ versus supine head rotated $90^{\circ}$ with the bed at $0^{\circ}$, Outcome 4 Cystic periventricular leukomalacia.

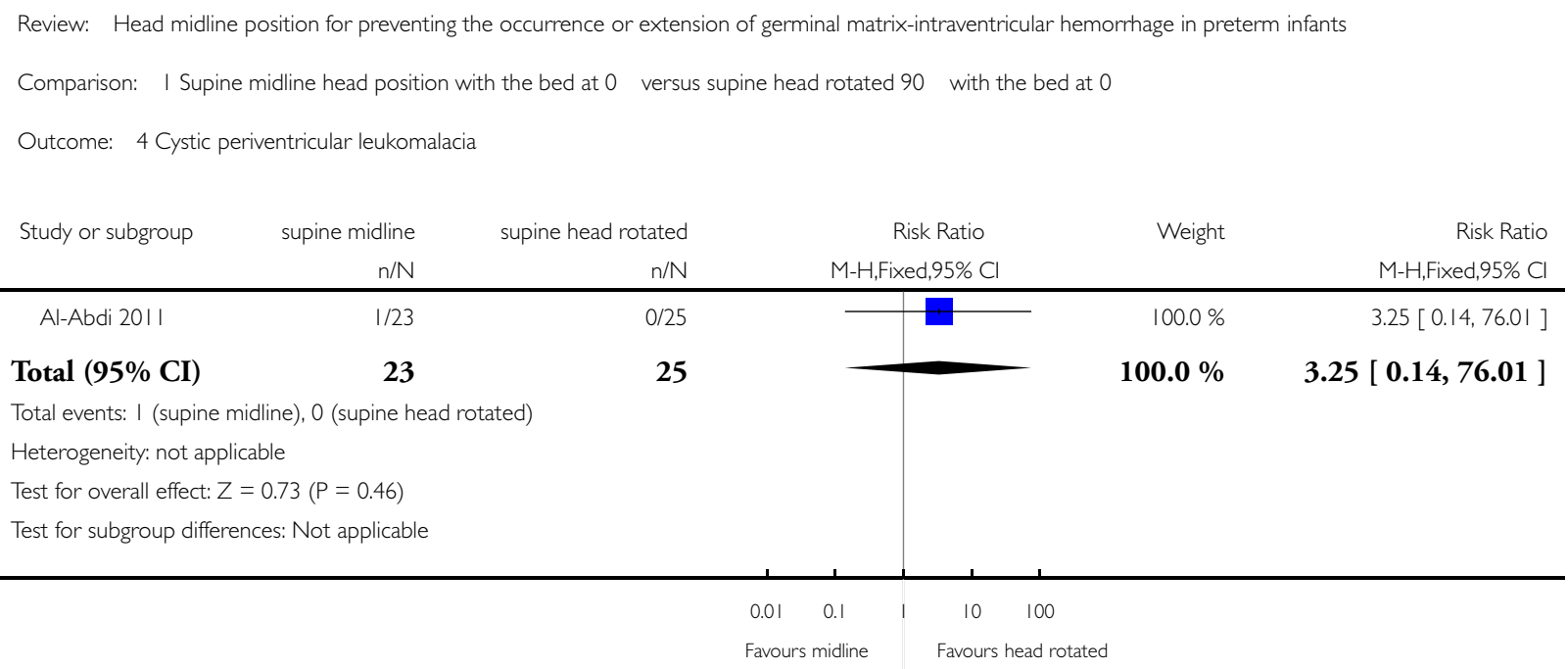


Analysis 1.5. Comparison I Supine midline head position with the bed at $0^{\circ}$ versus supine head rotated $90^{\circ}$ with the bed at $0^{\circ}$, Outcome 5 Retinopathy of prematurity, any stage.

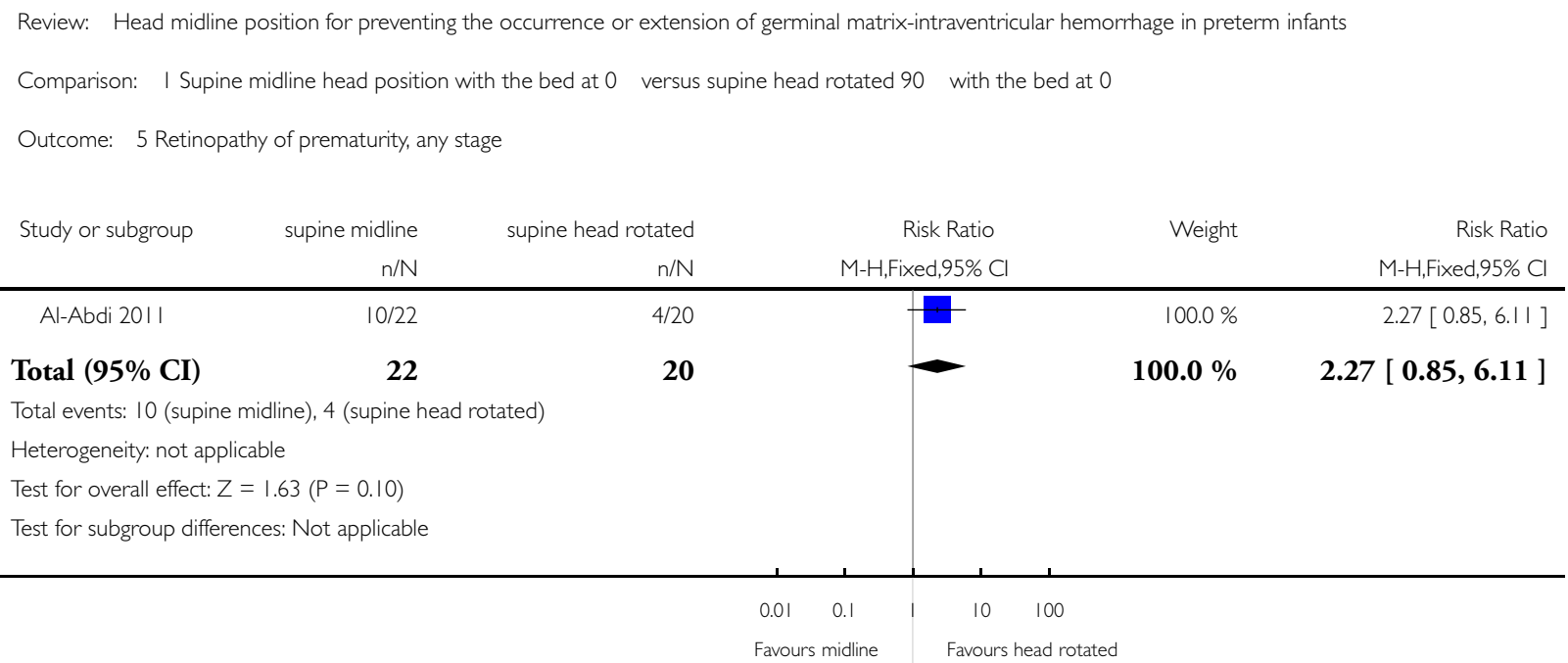

Analysis I.6. Comparison I Supine midline head position with the bed at $0^{\circ}$ versus supine head rotated $90^{\circ}$ with the bed at $0^{\circ}$, Outcome 6 Retinopathy of prematurity, $\geq$ stage 3 .

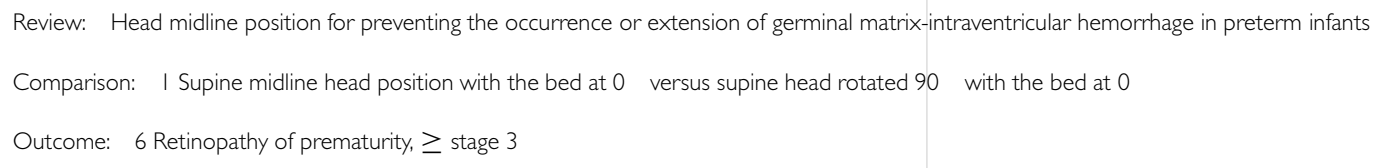

\begin{tabular}{|c|c|c|c|c|c|}
\hline \multirow[t]{2}{*}{ Study or subgroup } & supine midline & supine head rotated & Risk Ratio & Weight & Risk Ratio \\
\hline & $\mathrm{n} / \mathrm{N}$ & $\mathrm{n} / \mathrm{N}$ & M-H,Fixed,95\% Cl & & M-H,Fixed,95\% Cl \\
\hline Al-Abdi 201 I & $3 / 22$ & $1 / 20$ & & $100.0 \%$ & $2.73[0.31,24.14]$ \\
\hline Total (95\% CI) & 22 & 20 & & $100.0 \%$ & $2.73[0.31,24.14]$ \\
\hline \multicolumn{6}{|c|}{ Total events: 3 (supine midline), I (supine head rotated) } \\
\hline \multicolumn{6}{|c|}{ Heterogeneity: not applicable } \\
\hline \multicolumn{6}{|c|}{ Test for overall effect: $Z=0.90(P=0.37)$} \\
\hline Test for subgroup diff & Not applicable & & & & \\
\hline
\end{tabular}

$\begin{array}{cccc}0.01 \quad 0.1 & 1 & 10 \quad 100 \\ \text { Favours midline } & \text { Favours head rotated }\end{array}$




\title{
A P P E N D I C E S
}

\section{Appendix I. Standard search methods}

PubMed: ((infant, newborn[MeSH] OR newborn OR neonate OR neonatal OR premature OR low birth weight OR VLBW OR LBW or infan* or neonat*) AND (randomized controlled trial [pt] OR controlled clinical trial [pt] OR Clinical Trial[ptyp] OR randomized [tiab] OR placebo [tiab] OR clinical trials as topic [mesh: noexp] OR randomly [tiab] OR trial [ti]) NOT (animals [mh] NOT humans $[\mathrm{mh}]))$

Embase: (infant, newborn or newborn or neonate or neonatal or premature or very low birth weight or low birth weight or VLBW or LBW or Newborn or infan* or neonat*) AND (human not animal) AND (randomized controlled trial or controlled clinical trial or randomized or placebo or clinical trials as topic or randomly or trial or clinical trial)

CINAHL: (infant, newborn OR newborn OR neonate OR neonatal OR premature OR low birth weight OR VLBW OR LBW or Newborn or infan* or neonat*) AND (randomized controlled trial OR controlled clinical trial OR randomized OR placebo OR clinical trials as topic OR randomly OR trial OR PT clinical trial)

Cochrane Library: (infant or newborn or neonate or neonatal or premature or very low birth weight or low birth weight or VLBW or LBW)

\section{CONTRIBUTIONSOFAUTHORS}

$\mathrm{OR}$ and $\mathrm{MB}$ reviewed the literature and wrote the review.

MGC commented on and reviewed the review.

\section{DECLARATIONSOF INTEREST}

OR, MGC, and MB have no known conflicts of interest to declare.

\section{SOURCES OF SUPPORT}

\author{
Internal sources \\ - Institute for Clinical Sciences, Lund University, Lund, Sweden. \\ to $\mathrm{OR}$ and $\mathrm{MB}$ \\ - Istituto Giannina Gaslini, Genoa, Italy. \\ to MGC
}

\section{External sources}

- Eunice Kennedy Shriver National Institute of Child Health and Human Development, National Institutes of Health, Department of Health and Human Services, USA.

Editorial support of the Cochrane Neonatal Review Group has been funded with Federal funds from the Eunice Kennedy Shriver National Institute of Child Health and Human Development, National Institutes of Health, Department of Health and Human Services, USA, under Contract No. HHSN275201100016C 


\section{INDEX TERMS}

\section{Medical Subject Headings (MeSH)}

*Cerebral Ventricles; *Head; *Supine Position; Beds; Cerebral Hemorrhage [etiology; * prevention \& control]; Cerebrovascular Circulation [physiology]; Infant Mortality; Infant, Premature; Infant, Premature, Diseases [etiology; *prevention \& control]; Patient Positioning [*methods]; Publication Bias; Randomized Controlled Trials as Topic

\section{MeSH check words}

Humans; Infant; Infant, Newborn 